Supporting Information for

\title{
Effects of Ligand Shell Composition on Surface Reduction in PbS Quantum Dots
}

Carolyn L. Hartley , Melody L. Kessler, Christian Y. Dones Lassalle, Andrew M. Camp, and Jillian L. Dempsey*

Department of Chemistry, University of North Carolina, Chapel Hill, NC 27599-3290

*Correspondence to: dempseyi@email.unc.edu

Table of Contents $\quad$ Page

Additional experimental procedures ....................................................................... 2

TEM images and histograms for oleate-PbS QDs ................................................... 4

ATR-FTIR spectra of oleate-PbS and mixed-shell PbS QDs, and exchange ligands................. 5

TEM images and histograms for mixed-shell PbS QDs ......................................................... 9

XPS spectra and $\mathrm{Pb}: \mathrm{S}$ ratios of oleate-PbS and mixed-shell PbS QDs..................................... 11

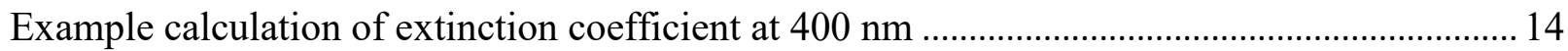

Calculated $\mathrm{Pb}$ and $\mathrm{S}$ values per QD using $\mathrm{Pb}: \mathrm{S}$ ratios from XPS ............................................ 16

DOSY NMR spectrum of toluate/oleate-PbS QD system .............................................. 17

${ }^{1} \mathrm{H}$ NMR characterization of oleate-PbS and mixed-shell PbS QD systems ............................ 18

DOSY NMR spectrum of UDT/oleate-PbS QD system .....................................................2

Ligand displacement and surface charging in oleate- $\mathrm{PbS}$ and mixed-shell $\mathrm{PbS}$ QDs monitored via

${ }^{1} \mathrm{H}$ NMR and UV-Vis with excess CoCp 2 added....................................................................23

Molecular orbital diagrams to illustrate $\mathrm{Pb}^{2+}$-ligand energetics.............................................. 45

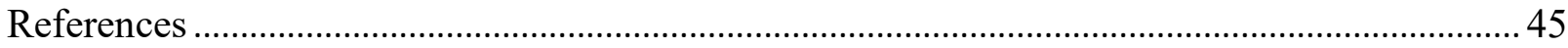




\section{Additional Experimental Procedures}

Details of Advanced NMR Methods and Relaxation Studies. Proton and relaxation studies were carried out either on a Bruker 600 Avance NEO spectrometer equipped with a cryoQNP cryoprobe or Bruker 600 Avance III equipped with a cryoQCI cryoprobe. All DOSY experiments were run on a Bruker 600 Avance system. Samples were thermoregulated at $25{ }^{\circ} \mathrm{C}$ and allowed to equilibrate 5 minutes before NMR acquisition. NMR processing was performed in MNOVA, and spectra were baseline corrected with a Whittaker smoother. While the exact baseline correction parameters vary from experiment to experiment, care was taken not to over-correct the broad QD features using large filters and smooth factors (exemplum: filter 111, smooth factor 16,384,000).

Table S1. Delay time d1 check with toluate ligands. $600 \mathrm{MHz}$ NMR, varied d1 delay time and used 12 scans for all. $46 \mu \mathrm{M}$ QD in toluene- $d_{8}$ with 1,3,5-trimethoxybenzene as internal standard.

\begin{tabular}{|c|c|c|c|}
\hline d1 delay time & bound toluate/QD & bound OA/QD & total toluate+OA ligands \\
\hline $\mathbf{3 0} \mathbf{~ s e c}$ & 53 & 73 & 126 \\
\hline $\mathbf{4 5} \mathbf{~ s e c}$ & 50 & 73 & 123 \\
\hline $\mathbf{6 0} \mathbf{~ s e c}$ & 48 & 73 & 121 \\
\hline $\mathbf{9 0} \mathbf{~ s e c}$ & 53 & 74 & 127 \\
\hline $\mathbf{1 2 0} \mathbf{s e c}$ & 53 & 73 & 128 \\
\hline $\mathbf{1 5 0} \mathbf{~ s e c}$ & 54 & 73 & \\
\hline
\end{tabular}

Preparation of lead (II) dodecanethiolate complex: Lead (II) dodecanethiolate was prepared following a modified literature procedure. ${ }^{1} \mathrm{~Pb}(\text { oleate })_{2}(76.4 \mathrm{mg}, 0.0992 \mathrm{mmol})$ and 1dodecanethiol $(60 \mu \mathrm{L}, 0.25 \mathrm{mmol})$ were combined with $10 \mathrm{~mL}$ toluene in a $50 \mathrm{~mL}$ round bottom flask equipped with a stir bar. The heterogeneous white solution stirred under nitrogen for 15 minutes before heating to reflux. The solution stirred under reflux for 40 minutes, after which point the heat was removed and the solution cooled to room temperature. The solution was allowed to continue to stir at room temperature overnight, during which the solution changed from white to yellow in color. The crude reaction product was transferred to three centrifuge tubes $(3 \mathrm{~mL} / \mathrm{tube})$ and centrifuged for 5 minutes at $8600 \mathrm{rpm}$. The resulting clear and colorless supernatant was discarded leaving a solid yellow precipitate. The solid was resuspended in toluene and then centrifuged again to isolate a fine yellow powder. The powder was dried under high vacuum and then characterized by XPS (Figure S14). Poor solubility in organic solvents inhibited characterization by NMR spectroscopy. 
Precipitation Procedure for Isolating Displaced Toluate from Reduced Toluate/Oleate-PbS QDs.

A $150 \mu \mathrm{M}$ solution $\left(600 \mu \mathrm{L}, 9 \cdot 10^{-8} \mathrm{~mol}\right)$ of toluate/oleate-PbS QDs in toluene- $d_{8}$ was prepared in an inert-atmosphere glovebox, and 500 eq. $\mathrm{CoCp}_{2}$ added $(300 \mu \mathrm{L}$ aliquot of a $150 \mathrm{mM}$ stock solution, $\left.4.5 \cdot 10^{-5} \mathrm{~mol}\right)$. The solution was allowed to react for four days under inert atmosphere shielded from light. A ${ }^{1} \mathrm{H}$ NMR spectrum of the sample was collected before precipitation (Figure S41). Inside of the glovebox, the sample was then divided between two $5 \mathrm{~mL}$ centrifuge tubes $(\sim 400-450 \mu \mathrm{L} /$ tube $)$ and filled to $5 \mathrm{~mL}$ total volume with acetonitrile to precipitate the QDs. The samples were then centrifuged at $9200 \mathrm{rpm}$ for 5 minutes, yielding a dark brown precipitate of QDs and a light brown supernatant. The precipitated QDs were dried under vacuum and then dispersed in toluene- $d_{8}$ for post-precipitation analysis (Figure S41). Portions of the resulting supernatant $(3 \mathrm{~mL})$ were transferred to two new centrifuge tubes and $2 \mathrm{~mL}$ acetonitrile added for a total volume of $5 \mathrm{~mL} /$ tube, and the samples centrifuged for 7 minutes. This second centrifugation produced a lightly colored brown-orange supernatant with a dark brown QD precipitate. The supernatant was transferred to a vial and solvent removed under vacuum, revealing a yellow-grey crystalline solid. The solid was redispersed in $10 \mathrm{~mL}$ of 1:1 acetone:acetonitrile, divided between two centrifuge tubes and centrifuged for 10 minutes, resulting in a small amount of dark brown precipitate. The supernatant from this final centrifugation step was transferred to a $20 \mathrm{~mL}$ vial and solvent removed under vacuum. The sample was then dispersed in acetonitrile- $d_{3}$ and analyzed by ${ }^{1} \mathrm{H}$ NMR spectroscopy (Figure S38). This precipitation procedure was repeated on a sample of toluate/oleate-PbS QDs (600 $\mu \mathrm{L}$, $\left.9 \cdot 10^{-8} \mathrm{~mol}\right)$ in toluene- $d_{8}$ without added $\mathrm{CoCp}_{2}$ to ensure that free toluate ligand was not displaced by the precipitation procedure ( $\sim 300 \mu \mathrm{L} /$ tube) (Figure $\mathbf{S 4 0}$ ). 

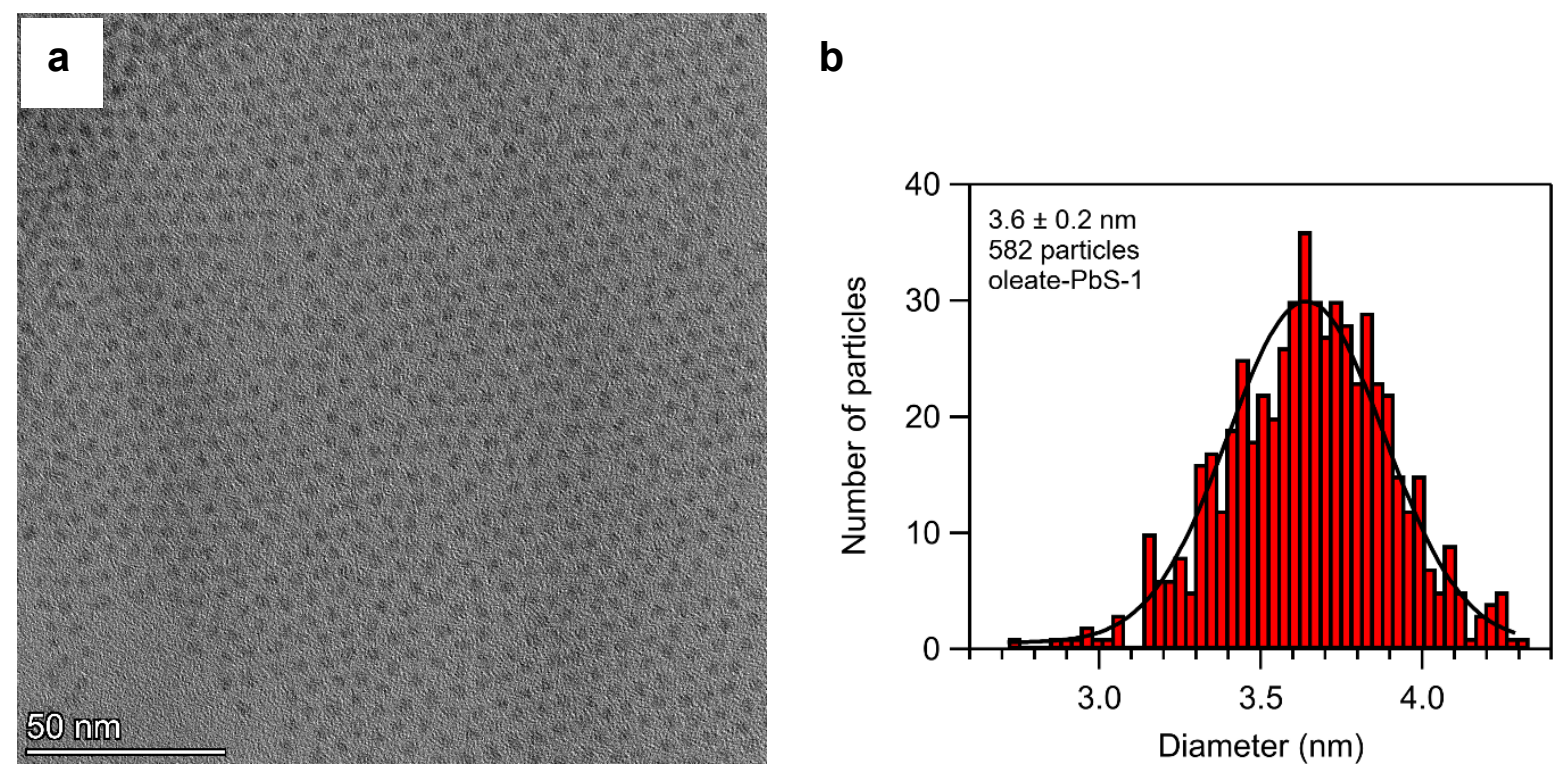

Figure S1. a) TEM image of as-synthesized oleate-PbS-1 used for comparison with System 1 (UDA/oleate-PbS QDs). b) Histogram constructed from analysis of the image in panel $a$ using ImageJ. Average particle size is $3.6 \pm 0.2 \mathrm{~nm}$.
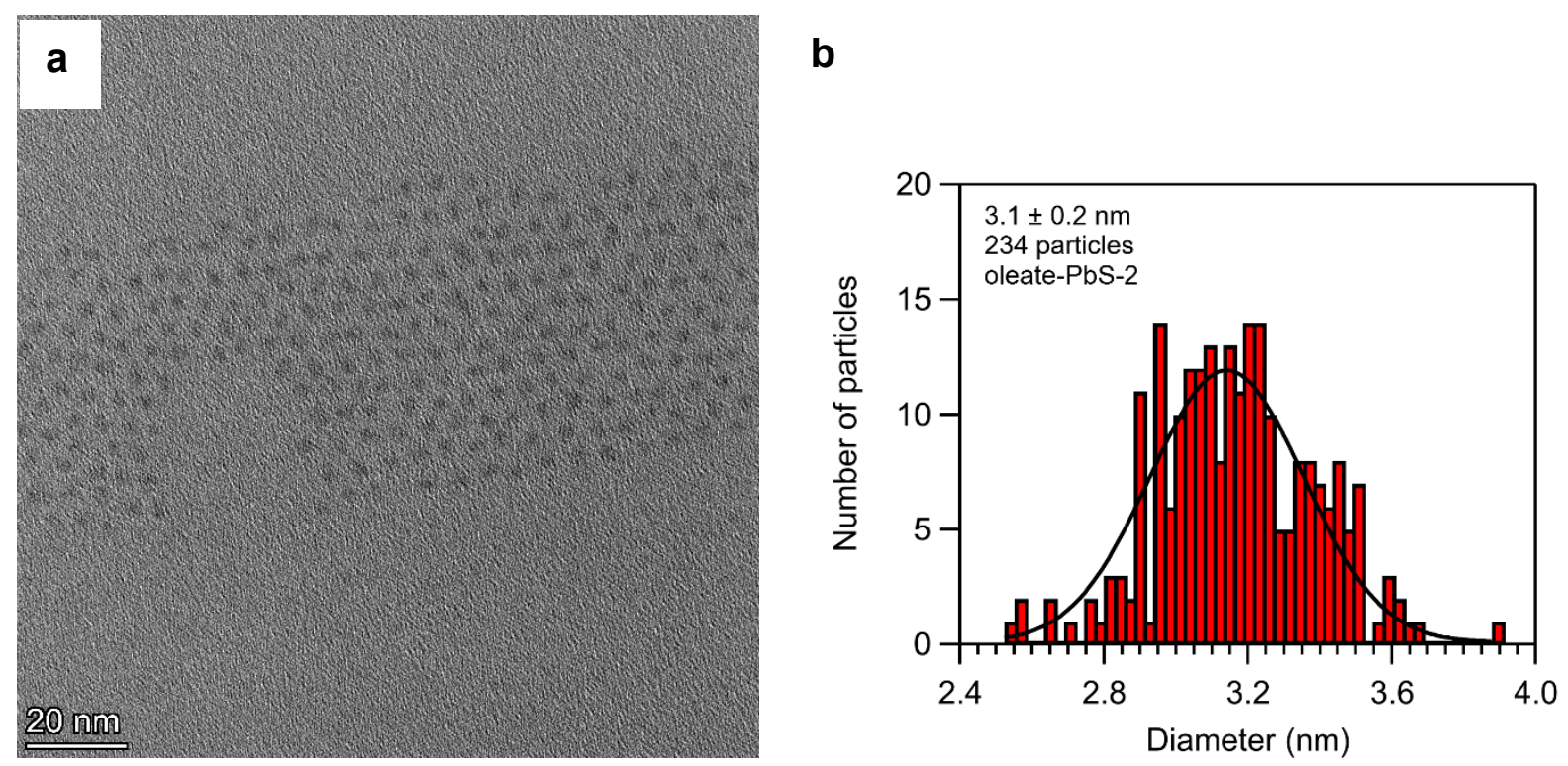

Figure S2. a) TEM image of as-synthesized oleate-PbS-2 used for comparison with System 2 (toluate/oleate-PbS QDs). b) Histogram constructed from analysis of the image in panel $a$ using ImageJ. Average particle size is $3.1 \pm 0.2 \mathrm{~nm}$. 


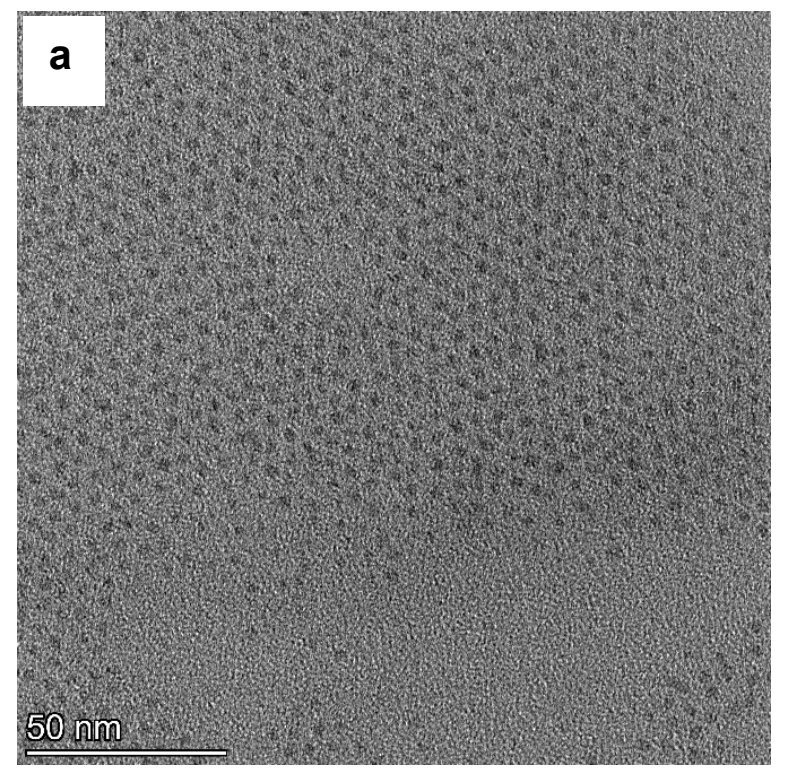

b

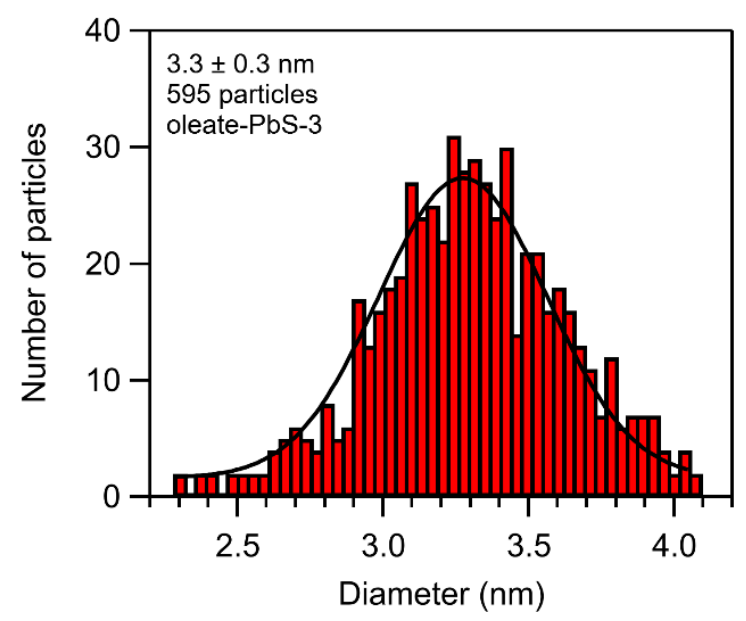

Figure S3. a) TEM image of as-synthesized oleate-PbS-3 used for comparison with System 3 (UDT/oleate-PbS QDs). b) Histogram constructed from analysis of the image in panel $a$ using ImageJ. Average particle size is $3.3 \pm 0.3 \mathrm{~nm}$.

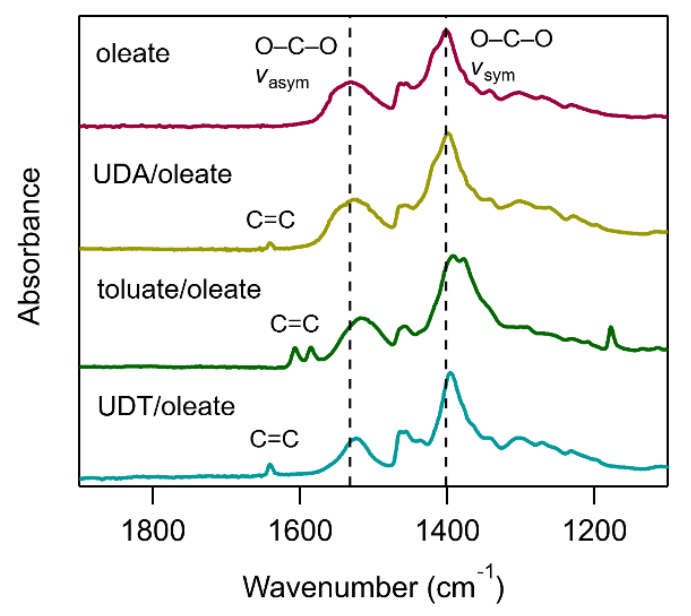

Figure S4. ATR-FTIR spectra of oleate-capped and mixed-shell QDs, zoomed in on the carboxylate stretching region. Dashed lines at 1532 and $1401 \mathrm{~cm}^{-1}$ are provided to enable qualitative comparisons of how the peak positions observed in the oleate- $\mathrm{PbS}$ spectrum shift in the mixed-shell spectra. In the UDA/oleate- $\mathrm{PbS}$ and UDT/oleate-PbS spectra, the appearance of a weak monosubstituted $\mathrm{C}=\mathrm{C}$ stretch at $1641 \mathrm{~cm}^{-1}$ consistent with the terminal alkene of UDA and UDT appear, confirming the ligand exchange. The conjugated $\mathrm{C}=\mathrm{C}$ stretching modes at $1607 \mathrm{~cm}^{-}$ ${ }^{1}$ and $1586 \mathrm{~cm}^{-1}$ observed in the toluate/oleate- $\mathrm{PbS}$ spectrum support the exchange of native oleates for toluate ligands. 


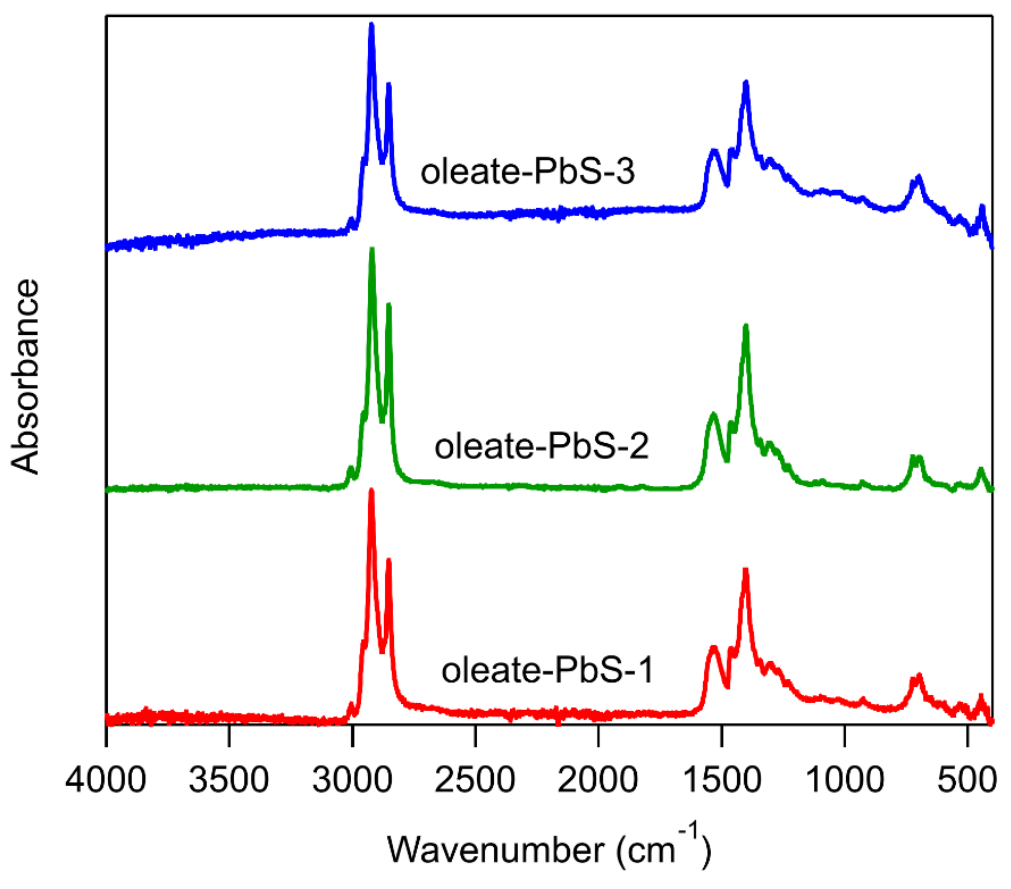

Figure S5. ATR-FTIR spectra of oleate-capped PbS QDs collected in absorbance mode. IR stretches are attributed to bound oleate ligands. 

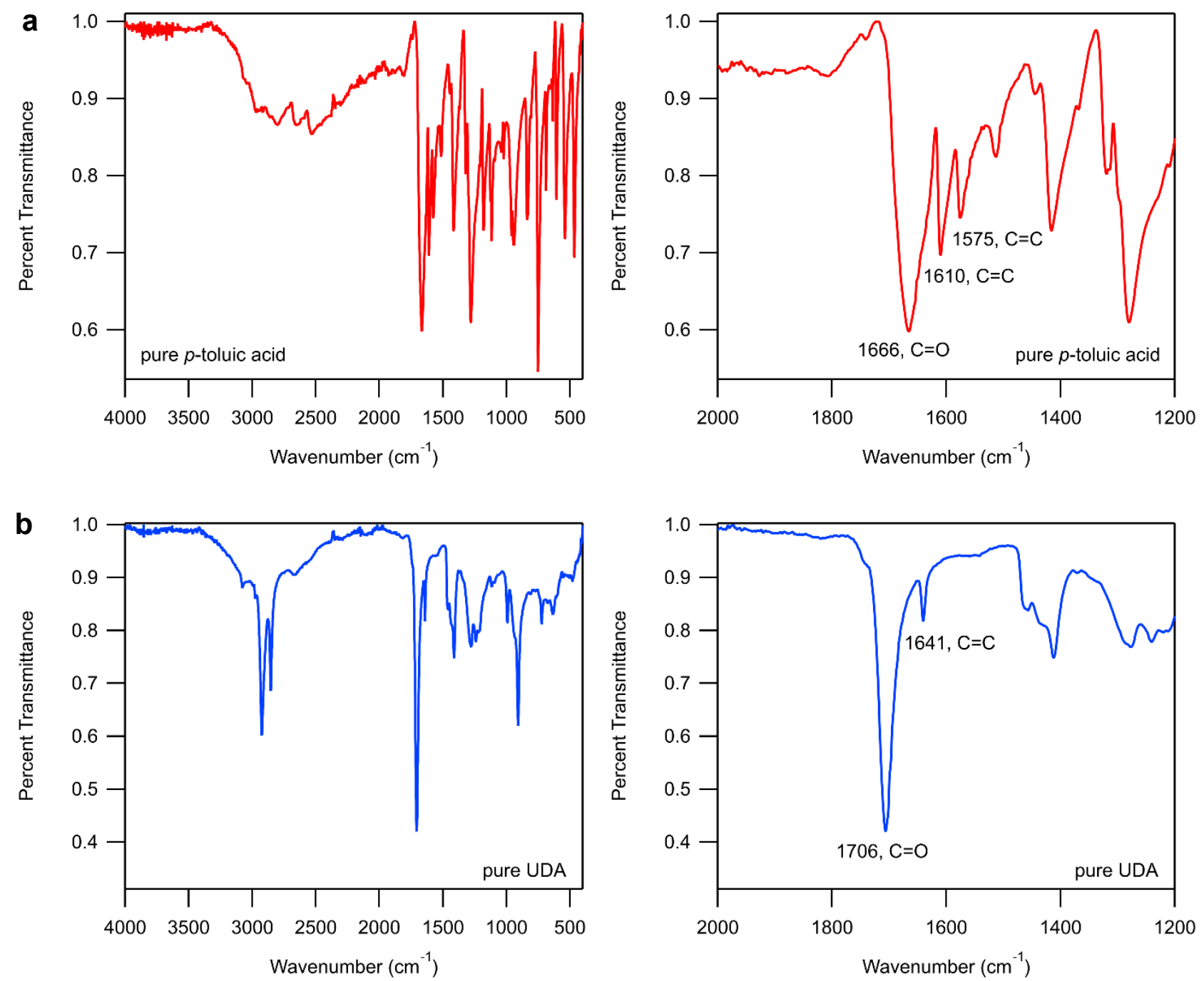

Figure S6. ATR-FTIR spectra of a) $p$-toluic acid and b) undec-10-enoic acid. Full spectra are provided on the left; zoomed-in spectra are shown on the right. The $\mathrm{C}=\mathrm{O}$ stretch of $p$-toluic acid appears at $1666 \mathrm{~cm}^{-1}$, and of UDA appears at $1706 \mathrm{~cm}^{-1}$. The labeled aryl $\mathrm{C}=\mathrm{C}$ peaks of $p$-toluic acid and alkene $\mathrm{C}=\mathrm{C}$ peaks of UDA are mostly unchanged upon binding to the $\mathrm{PbS}$ QD as described in the main text. 

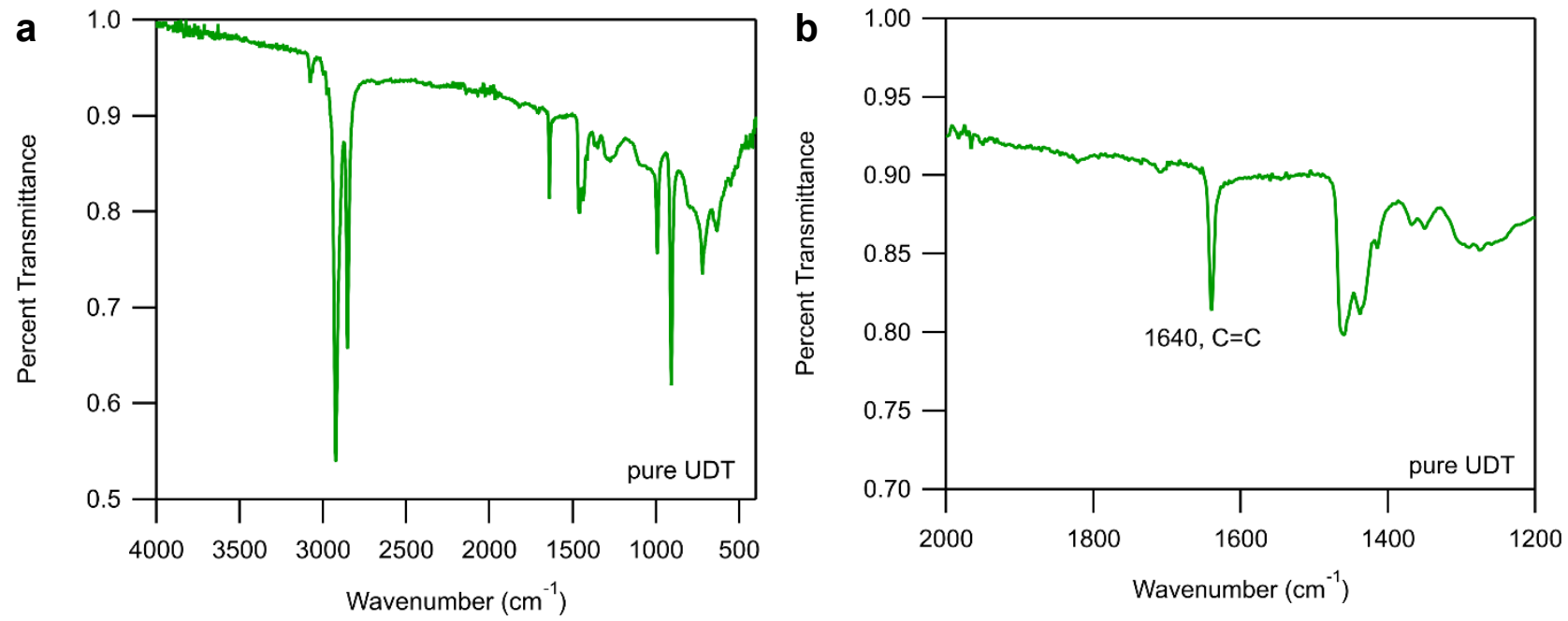

Figure S7. a) Full ATR-FTIR spectrum of undec-10-ene-1-thiol. b) Zoomed in spectrum of UDT, showing the $\mathrm{C}=\mathrm{C}$ stretch at $1640 \mathrm{~cm}^{-1}$, which appears unchanged in the spectrum of UDT/oleate-PbS QDs.

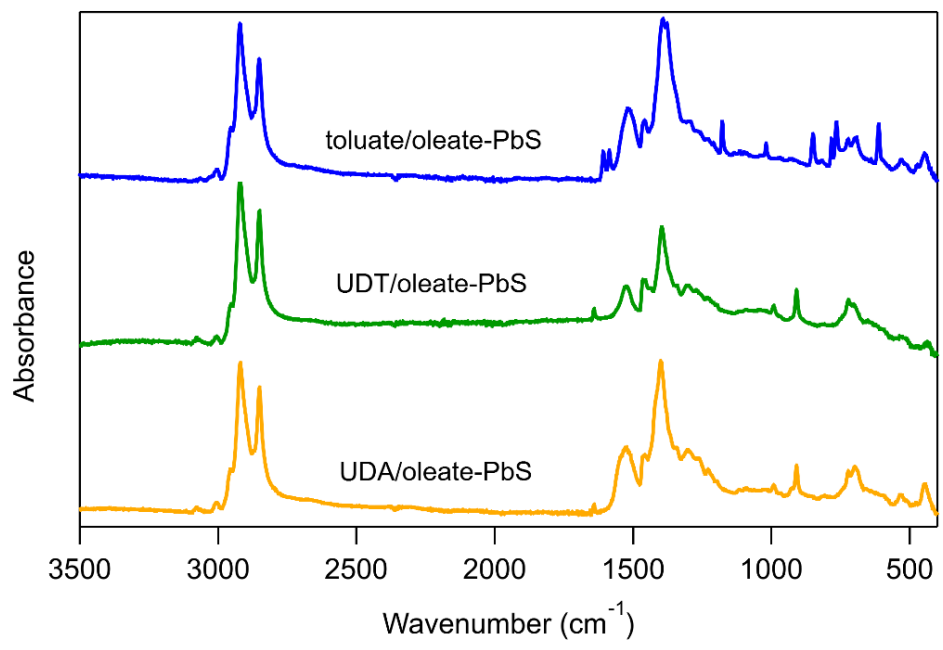

Figure S8. Full ATR-FTIR spectra for mixed-shell PbS QDs. 


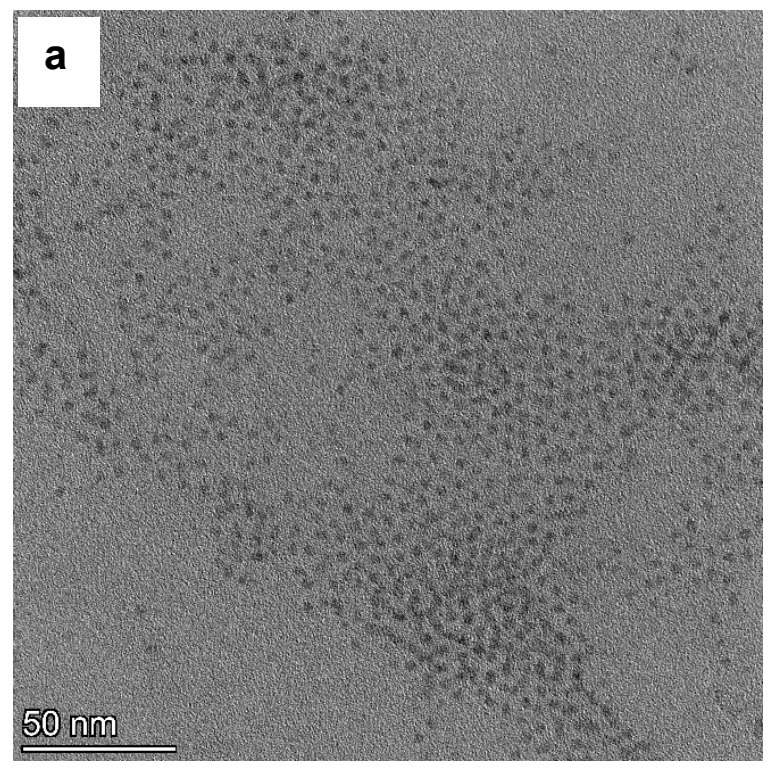

b

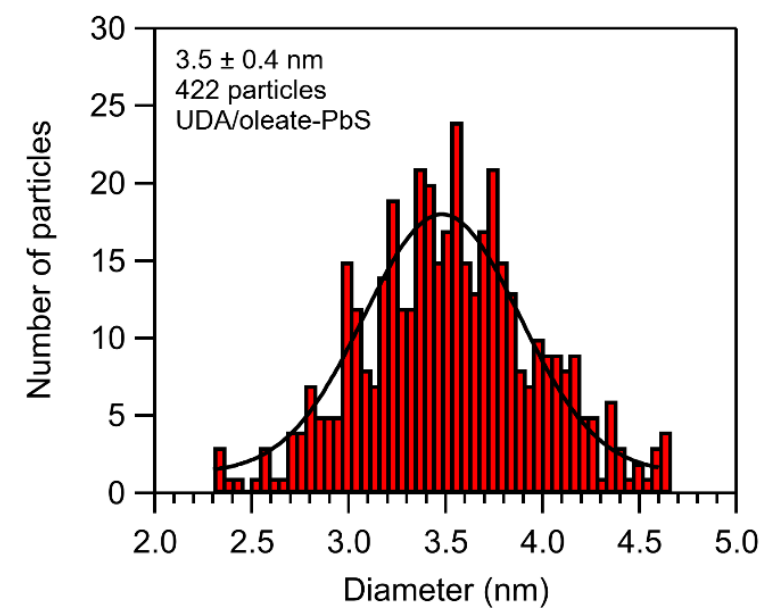

Figure S9. a) TEM image of exchanged and isolated UDA/oleate-PbS QDs (System 1). b) Histogram constructed from analysis of the image in panel a using ImageJ. Average particle size is $3.5 \pm 0.4 \mathrm{~nm}$.
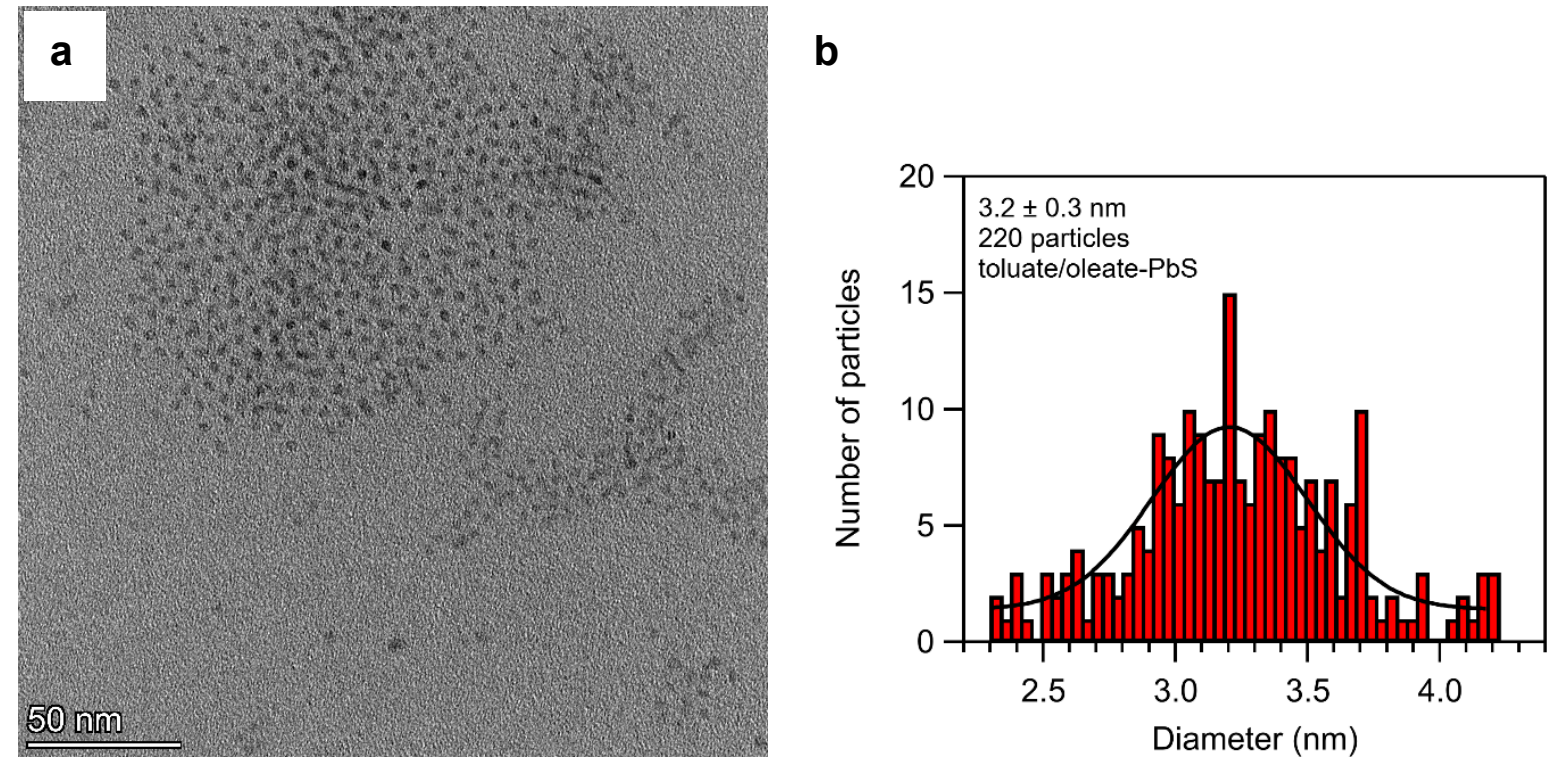

Figure S10. a) TEM image of exchanged and isolated toluate/oleate-PbS QDs (System 2). b) Histogram constructed from analysis of the image in panel a using ImageJ. Average particle size is $3.2 \pm 0.3 \mathrm{~nm}$. 


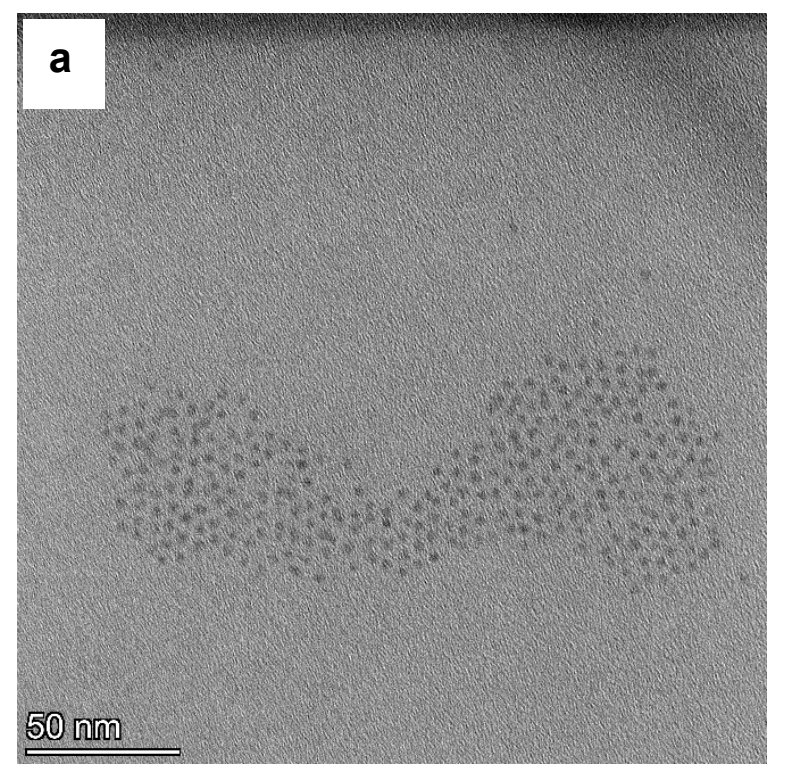

b

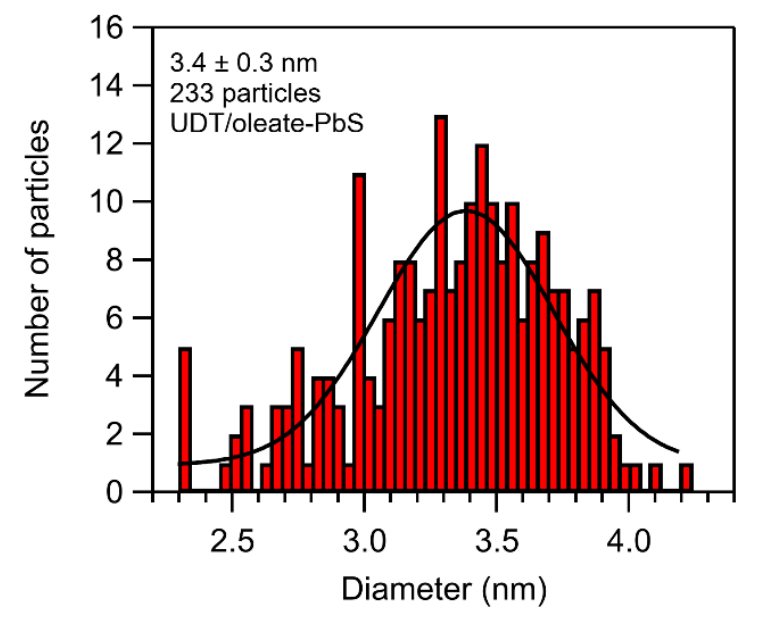

Figure S11. a) TEM image of exchanged and isolated UDT/oleate-PbS QDs (System 3). b) Histogram constructed from analysis of the image in panel $a$ using ImageJ. Average particle size is $3.4 \pm 0.3 \mathrm{~nm}$. 

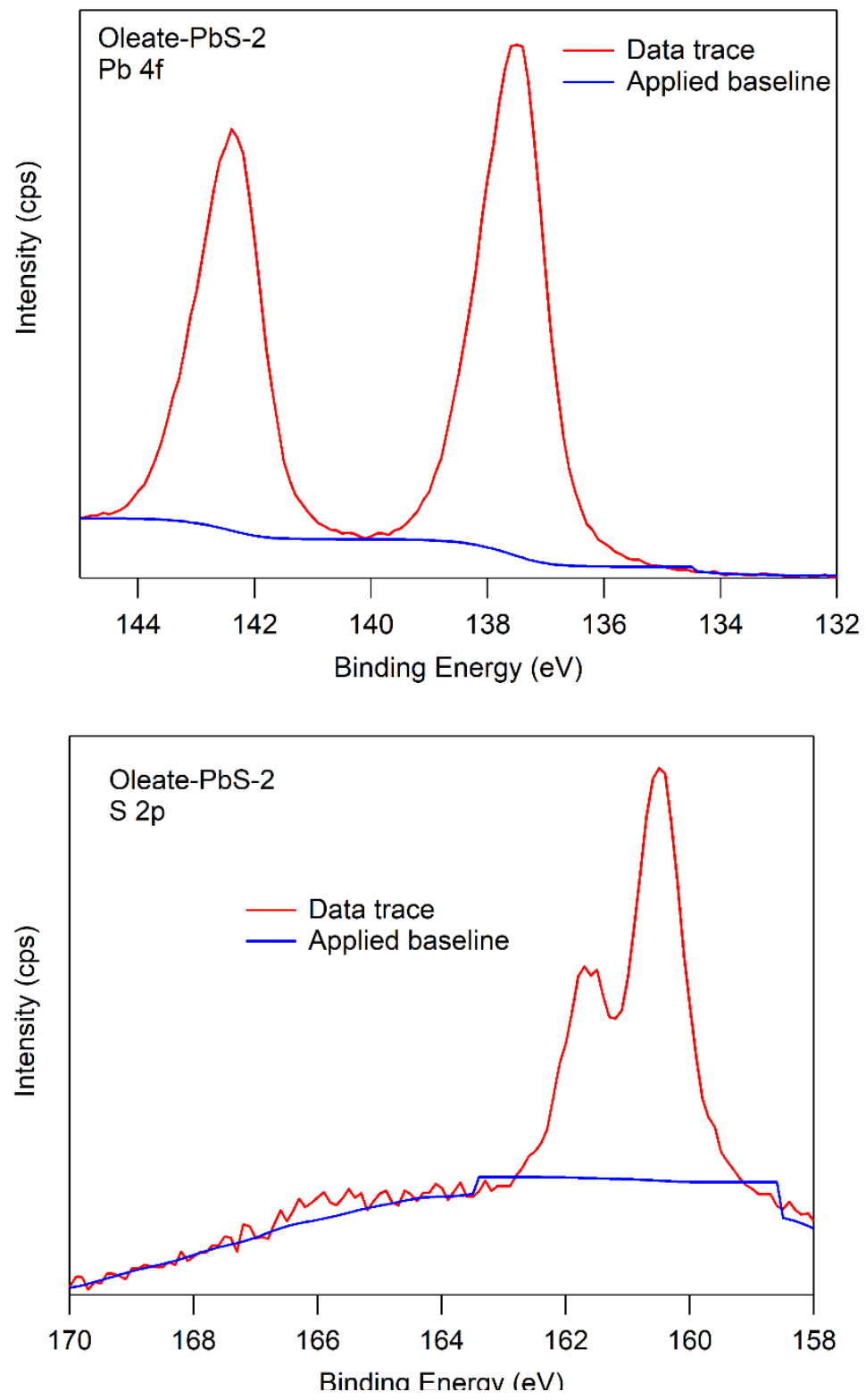

Figure S12. Representative XPS data of $\mathrm{Pb} 4 \mathrm{f}$ (top) and $\mathrm{S} 2 \mathrm{p}$ (bottom) regions for as-synthesized oleate-PbS-2 QDs. 
Table S2. Pb:S ratios for oleate-PbS QDs and their corresponding mixed-shell PbS QDs. The $\mathrm{Pb}: \mathrm{S}$ ratios were obtained from atomic concentrations of $\mathrm{Pb}$ and $\mathrm{S}$ determined by XPS. Two data points were obtained for two separate wafers by moving the wafer to examine a different spot on the surface.

\begin{tabular}{|c|c|c|c|c|c|c|c|}
\hline & & \multicolumn{6}{|c|}{$\mathrm{Pb}: \mathrm{S}$ ratios } \\
\hline \multicolumn{2}{|c|}{ XPS Sample } & $\begin{array}{l}\text { oleate- } \\
\text { PbS-1 }\end{array}$ & $\begin{array}{l}\text { UDA/oleate } \\
- \text { PbS }^{\mathbf{a}}\end{array}$ & $\begin{array}{l}\text { oleate- } \\
\text { PbS-2 }\end{array}$ & $\begin{array}{c}\text { toluate/oleate- } \\
\text { PbS }\end{array}$ & $\begin{array}{l}\text { oleate- } \\
\text { PbS-3 }\end{array}$ & $\begin{array}{c}\text { UDT/oleate- } \\
\text { PbS }\end{array}$ \\
\hline \multirow{2}{*}{$\begin{array}{c}\text { Wafer } \\
1\end{array}$} & 1 & 2.06 & 1.82 & 2.21 & 1.91 & 1.89 & 1.74 \\
\hline & 2 & 1.98 & - & 2.16 & 1.96 & 1.84 & 1.66 \\
\hline \multirow{2}{*}{$\begin{array}{c}\text { Wafer } \\
2\end{array}$} & 1 & 1.88 & 1.83 & 2.01 & 1.88 & 1.79 & 1.72 \\
\hline & 2 & 1.94 & 1.85 & 2.09 & 1.91 & 1.74 & 1.75 \\
\hline \multicolumn{2}{|c|}{$\begin{array}{c}\text { Average } \pm \\
\text { Std. Dev. }\end{array}$} & $\begin{array}{c}1.97 \pm \\
0.08\end{array}$ & $1.83 \pm 0.02$ & $\begin{array}{c}2.12 \pm \\
0.09\end{array}$ & $1.92 \pm 0.03$ & $\begin{array}{c}1.82 \pm \\
0.06\end{array}$ & $\begin{array}{c}1.72 \pm \\
0.04\end{array}$ \\
\hline
\end{tabular}

${ }^{a}$ Attempts to sample additional points on wafer 1 for UDA/oleate-PbS resulted in charging. Point 2 was therefore omitted.

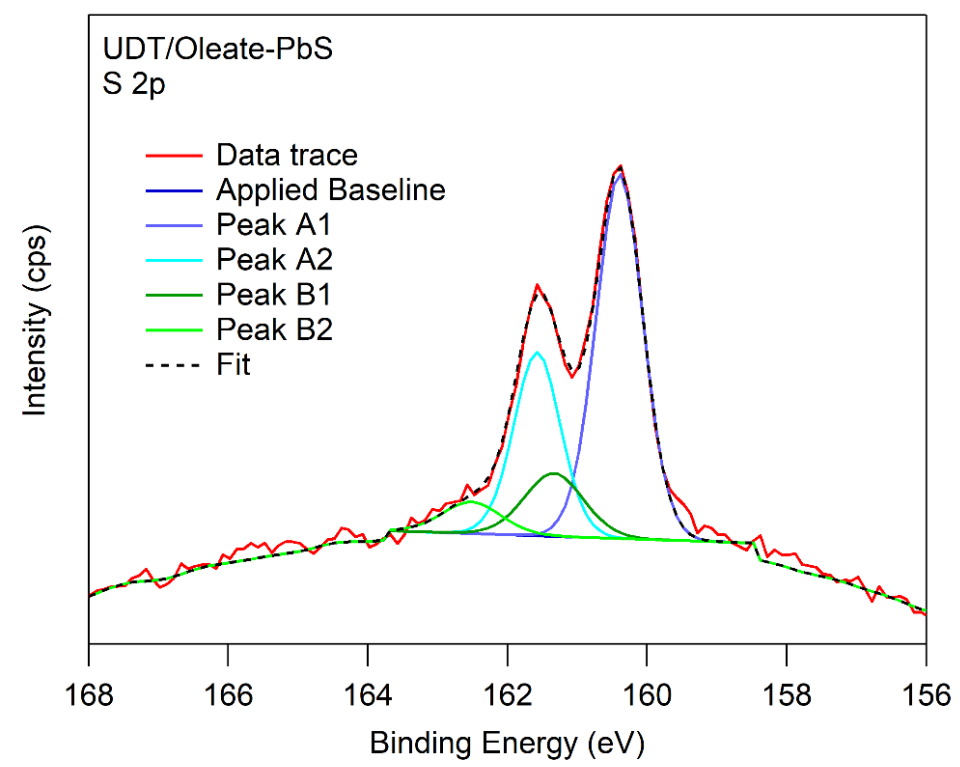

Figure S13. Representative S 2p region of XPS spectrum for UDT/oleate-PbS QDs. The doublet positioned at $160.4 \mathrm{eV}$ (Peaks A1 and A2, 160.4 and $161.6 \mathrm{eV}$, respectively) is attributed to sulfur in the nanocrystalline PbS lattice while the doublet at $161.3 \mathrm{eV}$ (Peaks B1 and B2, 161.3 and 162.5 $\mathrm{eV}$, respectively) is attributed to $\mathrm{Pb}$-bound thiolate ligands. A linear baseline was applied to the $\mathrm{S}$ $2 \mathrm{p}$ regions of $3.1 \mathrm{~nm}$ oleate- $\mathrm{PbS}$ and the exchanged UDT/oleate- $\mathrm{PbS}$ samples to standardize fitting parameters and enable comparison of $\mathrm{Pb}: \mathrm{S}$ ratios for these samples. A dwell time of $10000 \mathrm{~ms}$ was used improve resolution of S $2 p$ feature. 


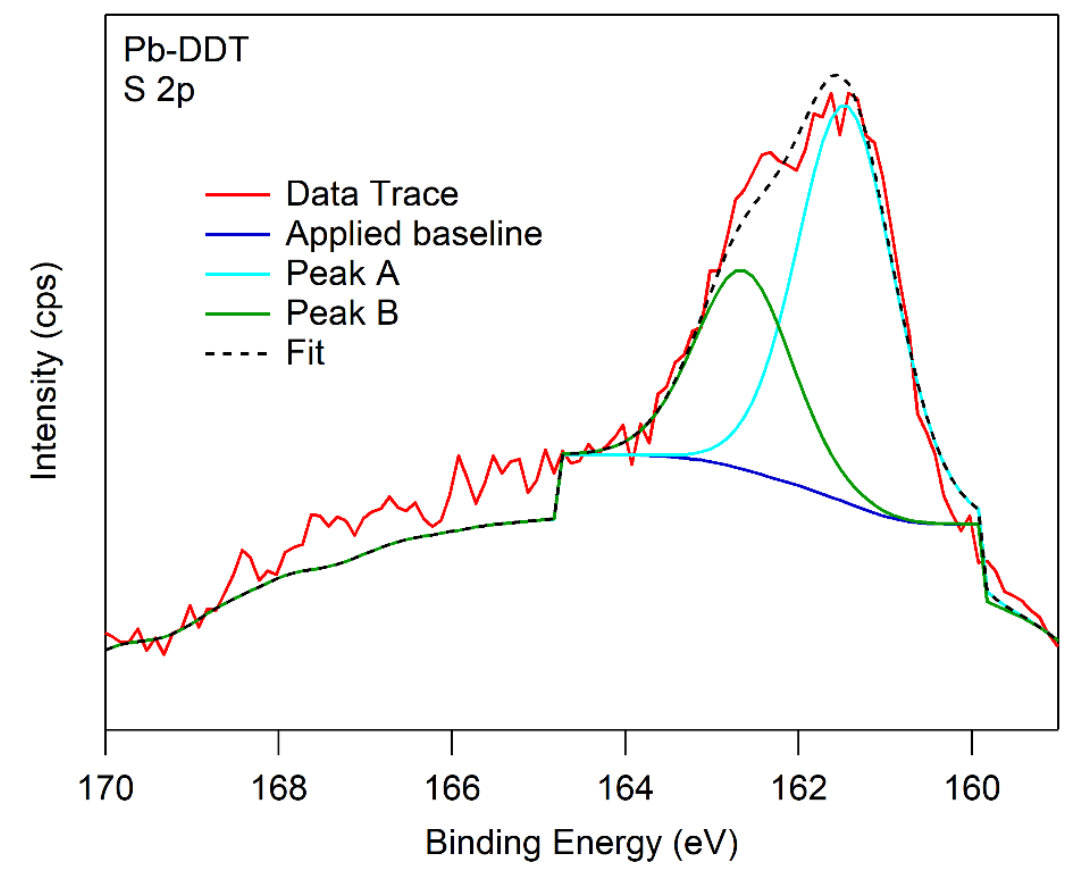

Figure S14. S $2 p$ region of XPS spectrum for putative Pb-DDT complex. The spectrum is fit well by underlying features at $161.5 \mathrm{eV}$ (Peak A) and $162.6 \mathrm{eV}$ (Peak B). A dwell time of $10000 \mathrm{~ms}$ was used improve resolution of $\mathrm{S} 2 \mathrm{p}$ feature. 


\section{Example Calculation of Extinction Coefficient at $400 \mathrm{~nm}$}

First, a sample of QDs with known volume $(600 \mu \mathrm{L})$ but unknown concentration was prepared in a $2 \mathrm{~mm}$ cuvette. Although the concentration was unknown, rough calculations of the anticipated QD concentration allowed for preparation of a sample for UV-Vis and ICP-MS analysis. The QD concentrations selected in this study both i) provide absorbance spectra with observable excitonic peaks and ii) allow for few serial dilutions to achieve $\mathrm{Pb}$ concentrations appropriate for ICP-MS.

A UV-Visible absorbance spectrum was collected and the A400 value was determined as shown:

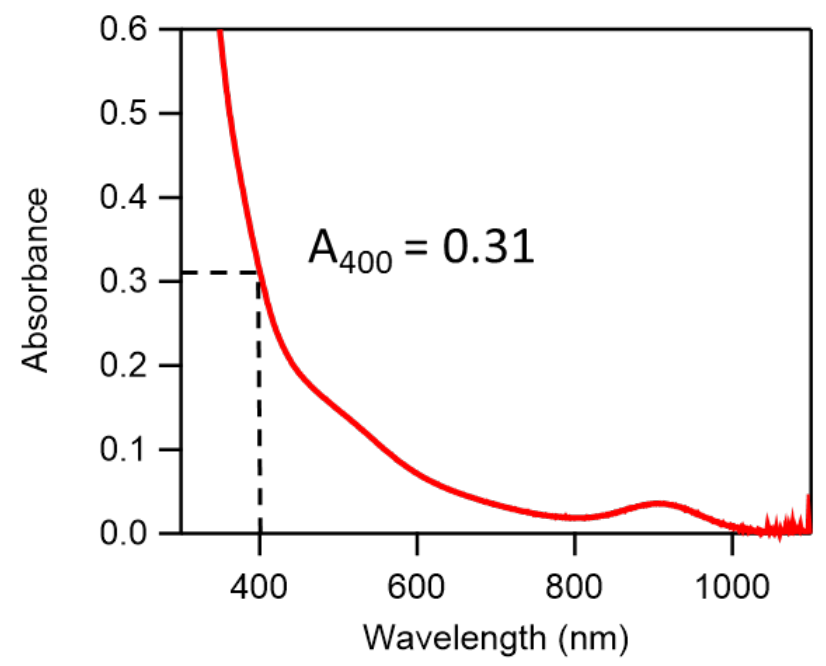

The contents of the cuvette were carefully and completely rinsed with pentane into a $20 \mathrm{~mL}$ scintillation vial and allowed to evaporate in a fume hood under air stream (previously, rotary evaporation had caused the sample to bump, which disrupted quantitative transfer and analysis of the sample). Next, the sample was pyrolyzed in the same vial in a box furnace at $450{ }^{\circ} \mathrm{C}$ for 30 min and allowed to cool to room temperature. Then, $0.5 \mathrm{~mL}$ of TraceMetals grade $\mathrm{HNO}_{3}$ was added to the sample and allowed to digest for a minimum of 4 hours. The sample was diluted with Millipore water and filtered through a pipette filter into a $10 \mathrm{~mL}$ volumetric flask to remove insoluble pyrolyzed organic particulates. The sample was transferred to a $15 \mathrm{~mL}$ centrifuge tube, and serial dilutions were performed to attain a concentration $250 \times$ more dilute than the initial sample. $\mathrm{Pb}$ concentrations of the $250 \times$ diluted samples were measured between 30 and $60 \mathrm{ppb} \mathrm{Pb}$ by ICP-MS.

The sample given in the UV-Vis spectrum above is from the oleate-PbS-2 QD batch, and had a Pb concentration of $42 \mathrm{ppb}$. Since this sample had been diluted by $250 \times$, the product of $42 \times 250$ yields the $\mathrm{Pb}$ concentration of the initial sample prepared in the $10 \mathrm{~mL}$ volumetric flask, $1.0 \cdot 10^{4} \mathrm{ppb} \mathrm{Pb}$. Converting from $\mathrm{ppb} \mathrm{Pb}$ to moles of $\mathrm{Pb}$ yields $5.1 \cdot 10^{-7}$ mol total $\mathrm{Pb}$, as follows:

$$
\begin{aligned}
1.0 \cdot 10^{4} \mathrm{ppb} & =\frac{1.0 \cdot 10^{4} \mu \mathrm{g}}{1 \mathrm{~L}} * \frac{1 \mathrm{~g}}{10^{6} \mu \mathrm{g}} * \frac{1 \mathrm{~mol} \mathrm{~Pb}}{207.2 \mathrm{~g}} * \frac{1 \mathrm{~L}}{1000 \mathrm{~mL}} * 10 \mathrm{~mL} \\
& =5.1 \cdot 10^{-7} \mathrm{~mol} \mathrm{~Pb} \text { in volumetric flask }
\end{aligned}
$$


Using Equation 2 in the main text gives the total number of ions in each QD:

$$
\mathrm{N}=\frac{4 \pi}{3}\left(\frac{2.9}{0.593}\right)^{3}=493 \text { total number of ions per QD }
$$

$\mathrm{The} \mathrm{Pb}: \mathrm{S}$ ratio taken from Table $\mathbf{S 2}$ for this set of QDs is $2.12 \mathrm{~Pb}: \mathrm{S}$. This is used to solve for one variable, $\mathrm{Pb}_{\text {total }}$ :

$$
\begin{aligned}
2.12 & =\frac{\mathrm{Pb}_{\text {total }}}{\mathrm{S}_{\text {total }}} \\
\mathrm{Pb}_{\text {total }} & =2.12 \mathrm{~S}_{\text {total }}
\end{aligned}
$$

The equation for total atoms $(\mathrm{N})$ is the sum of the total number of $\mathrm{Pb}$ ions $\left(\mathrm{Pb}_{\text {total }}\right)$ and the total number of $\mathrm{S}$ ions ( $\left.\mathrm{S}_{\text {total }}\right)$, which is not a 1:1 stoichiometry but depends on the $\mathrm{Pb}: \mathrm{S}$ ratio:

$$
\mathrm{Pb}_{\text {total }}+\mathrm{S}_{\text {total }}=\mathrm{N}
$$

Substituting $2.12 \mathrm{~S}_{\text {total }}$ into the equation for total number of $\mathrm{Pb}$ ions allows one to solve for total number of $\mathrm{S}$ ions:

$$
\begin{gathered}
2.12 \mathrm{~S}_{\text {total }}+1 \mathrm{~S}_{\text {total }}=\mathrm{N} \\
3.12 \mathrm{~S}_{\text {total }}=493 \\
\mathrm{~S}_{\text {total }}=158
\end{gathered}
$$

Next, total $\mathrm{Pb}$ per QD is calculated by subtracting $\mathrm{S}_{\text {total }}$ from $\mathrm{N}$ :

$$
493-158=335 \mathrm{~Pb}
$$

The total numbers of $\mathrm{Pb}$ and $\mathrm{S}$ ions per $\mathrm{QD}$ are given in Table $\mathbf{S 3}$ for all batches.

The next step in the calculation of the concentration of QDs in the cuvette is to combine the molar ratio of $335 \mathrm{~Pb}$ ions per $\mathrm{QD}$ with the total moles of $\mathrm{Pb}$ determined via ICP-MS:

$$
5.1 \cdot 10^{-7} \mathrm{~mol} \mathrm{~Pb} \times \frac{1 \mathrm{~mol} \mathrm{QD}}{335 \mathrm{~mol} \mathrm{~Pb}} \times \frac{1}{0.0006 \mathrm{~L}}=2.5 \cdot 10^{-6} \mathrm{M}=\mathrm{c}
$$

With the concentration of QDs, $2.5 \cdot 10^{-6} \mathrm{M}$, and the $\mathrm{A}_{400}$ value of 0.31 from the UV-Vis spectrum above, Beer's law is finally employed to determine the molar extinction coefficient at $400 \mathrm{~nm}$ :

$$
\varepsilon_{400}=\frac{\mathrm{A}_{400}}{\mathrm{bc}}=\frac{0.31}{(0.2 \mathrm{~cm}) \times\left(2.5 \cdot 10^{-6} \mathrm{M}\right)}=6.2 \cdot 10^{5} \mathrm{M}^{-1} \mathrm{~cm}^{-1}
$$


Table S3. $\mathrm{Pb}: \mathrm{S}$ ratios from XPS, calculated total ions per $\mathrm{QD}$, and calculated total $\mathrm{Pb}$ and total $\mathrm{S}$ ions per $\mathrm{QD}$ using the $\mathrm{Pb}: \mathrm{S}$ ratios.

\begin{tabular}{|c|c|c|c|c|c|}
\hline \multirow{2}{*}{ System } & Sample & $\begin{array}{c}\text { Average Pb:S ratio } \\
\pm \mathbf{S D}\end{array}$ & $\begin{array}{c}\text { Total Pb+S } \\
(\mathbf{N})^{\mathbf{a}}\end{array}$ & $\begin{array}{c}\text { Pb per } \\
\text { QD }\end{array}$ & $\begin{array}{c}\text { S per } \\
\text { QD }\end{array}$ \\
\hline \multirow{2}{*}{1} & Oleate-PbS-1 & $1.97 \pm 0.08$ & 681 & 451 & 230 \\
\cline { 2 - 6 } & UDA/oleate-PbS & $1.83 \pm 0.02$ & 681 & 441 & 240 \\
\hline \multirow{2}{*}{2} & & & & & \\
\cline { 2 - 7 } & Oleate-PbS-2 & $2.12 \pm 0.09$ & 493 & 335 & 158 \\
\hline \multirow{2}{*}{3} & Toluate/oleate-PbS & $1.92 \pm 0.03$ & 493 & 324 & 169 \\
\cline { 2 - 7 } & & & & & \\
\cline { 2 - 7 } & Oleate-PbS-3 & $1.82 \pm 0.06$ & 597 & 385 & 212 \\
\hline
\end{tabular}

${ }^{\mathrm{a} C a l c u l a t e d}$ per Equation 2 in the main text 


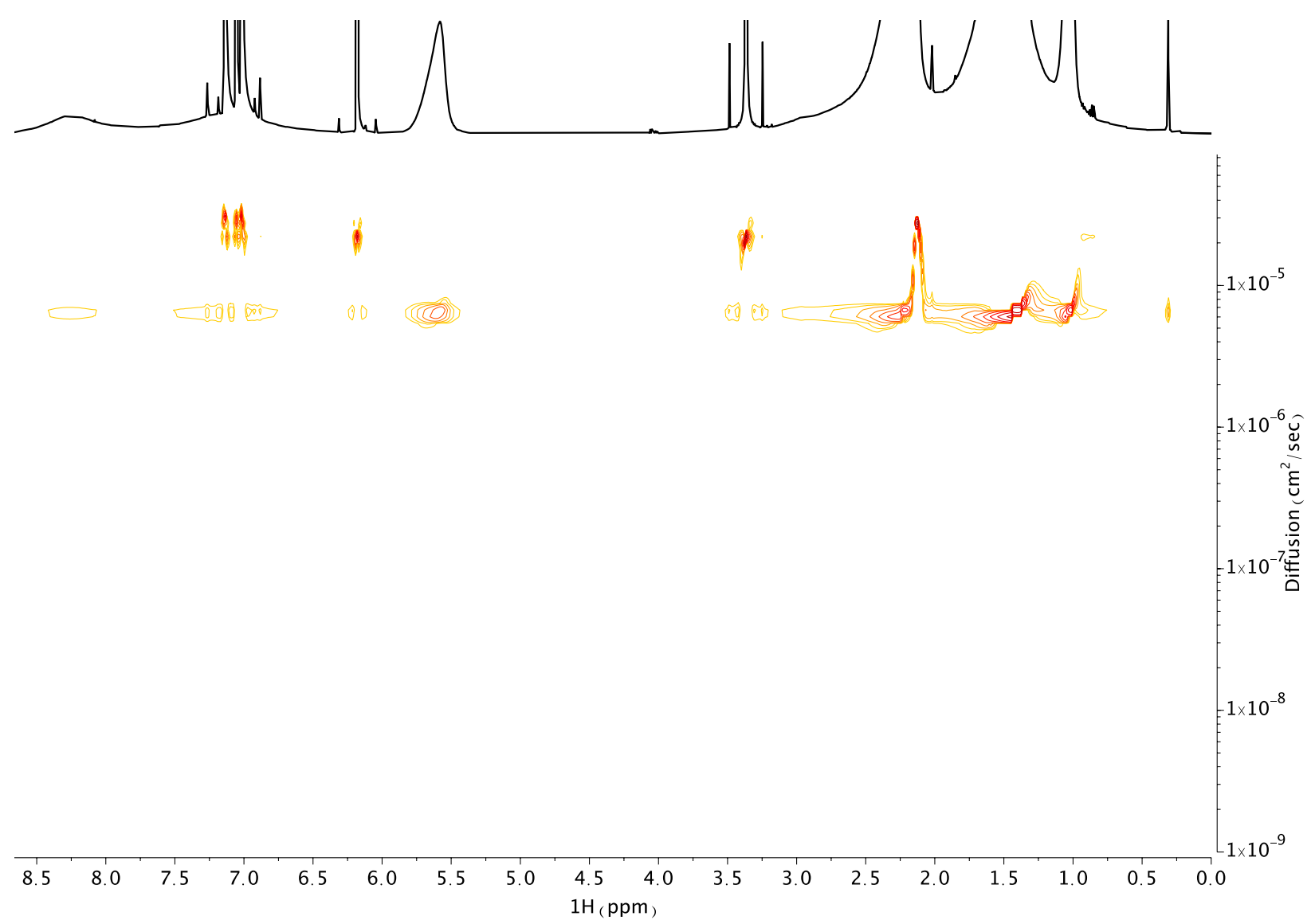

Figure S15. ${ }^{1} \mathrm{H}$ DOSY plot of the toluate/oleate-PbS QD system (150 $\mu \mathrm{M}$ in toluene- $\left.d_{8}\right)$. An optimized average $90^{\circ}$ pulse length of 15.125 microseconds and a relaxation delay of 15 seconds were used for this experiment. A stebpgp1s pulse sequence was used to maximize signal to noise. A delta of $100 \mathrm{~ms}$ and a gradient pulse length $(1100 \mathrm{msec})$ were used. An array of 1D spectra at 32 different gradient strengths with linear sampling were acquired and transformed using a Bayesian transformation in the MNOVA software suite (rf: 2, repetitions: 1). Analysis of the diffusion data reveals an estimated diffusion constant for the trimethoxybenzene internal standard of $2.2 \cdot 10^{-5} \mathrm{~cm}^{2} / \mathrm{sec}$. The toluate and oleate ligands show an estimated diffusion constant of $6.3 \cdot 10^{-}$ ${ }^{6} \mathrm{~cm}^{2} / \mathrm{sec}$, indicating that all ligand resonances are broadened due to heterogeneous broadening and not due to a convolution of bound and free ligand. Additionally, the smaller diffusion constant for the ligand resonances relative to that of the internal standard indicates that they are bound to the QD surface. 


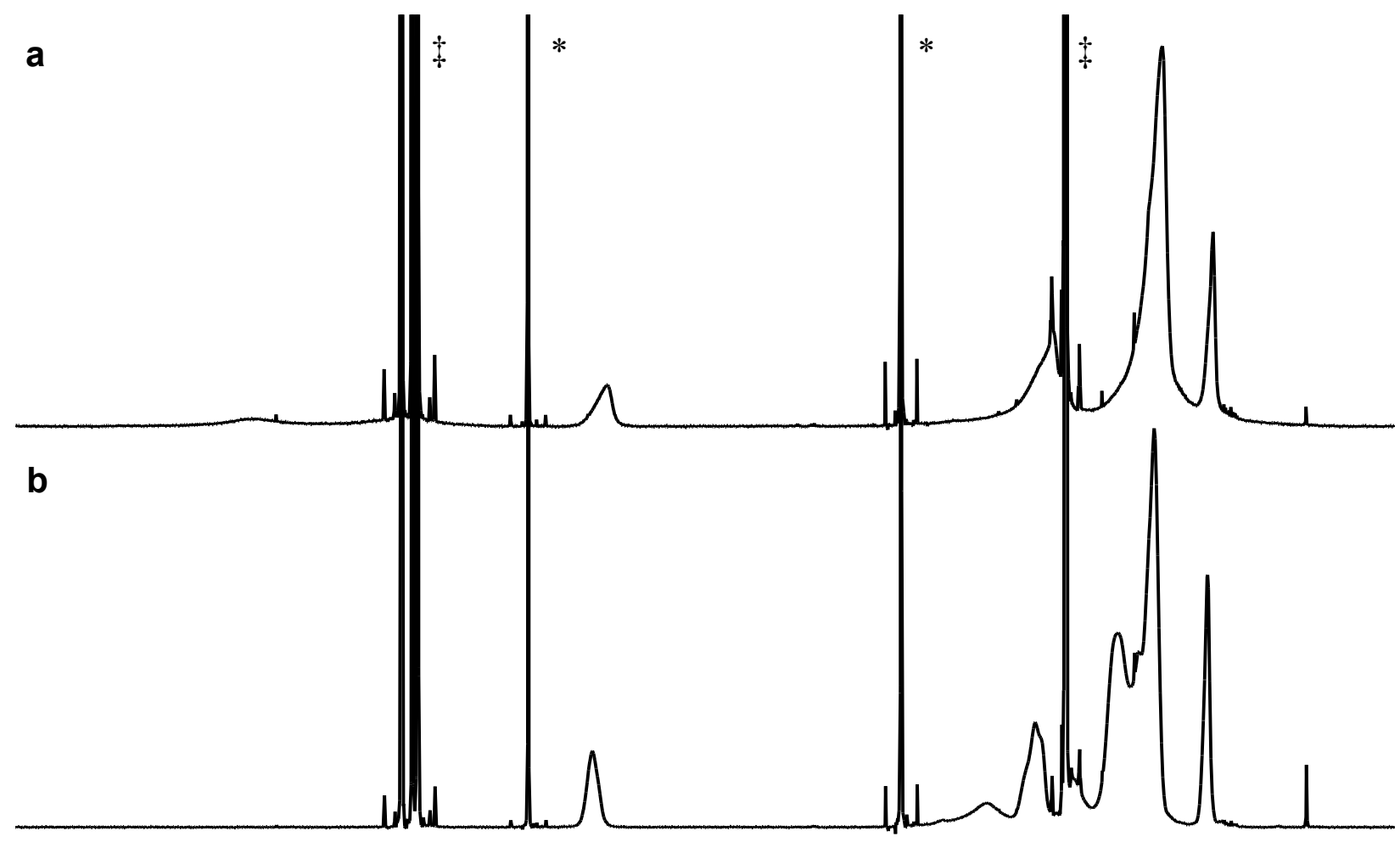

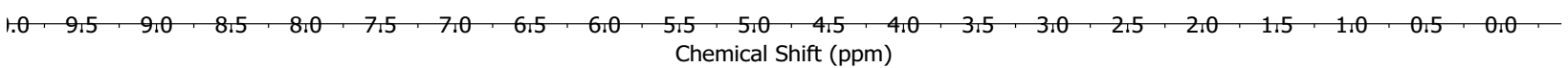

Figure S16. $600 \mathrm{MHz}{ }^{1} \mathrm{H}$ NMR spectra in toluene- $d_{8}$ of a) toluate/oleate-PbS QDs and b) $2.9 \mathrm{~nm}$ oleate-PbS QDs. Sharp peaks denoted by $\$$ are toluene solvent residuals, and peaks marked by * are protons within the internal standard 1,3,5-trimethoxybenzene. 

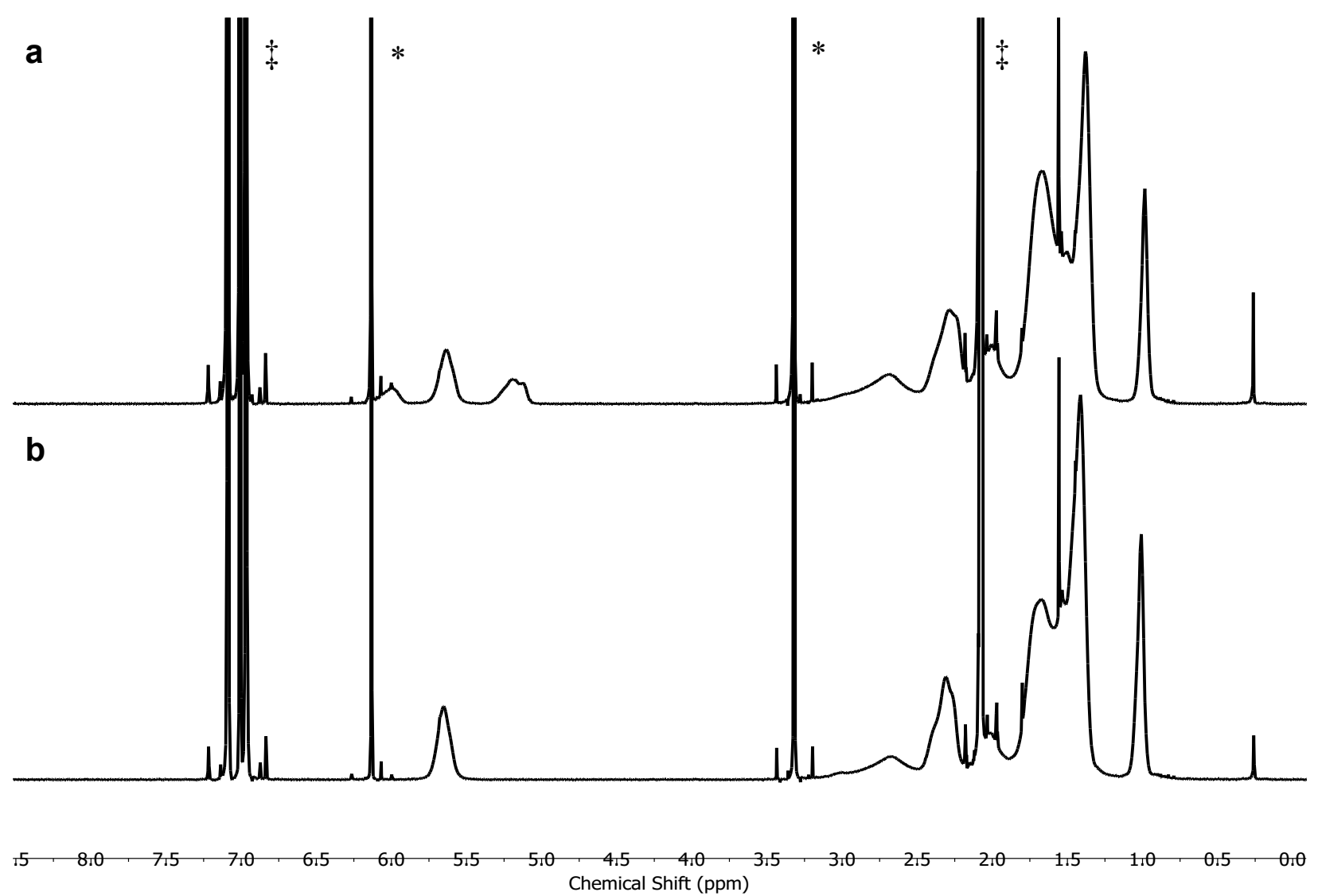

Figure S17. $600 \mathrm{MHz}{ }^{1} \mathrm{H}$ NMR spectra in toluene- $d_{8}$ of a) UDA/oleate-PbS QDs and b) $3.1 \mathrm{~nm}$ oleate-PbS QDs. Sharp peaks denoted by $\$$ are toluene solvent residuals, and peaks marked by * are protons within the internal standard 1,3,5-trimethoxybenzene. The impurity at $1.57 \mathrm{ppm}$ is trace acetone. 


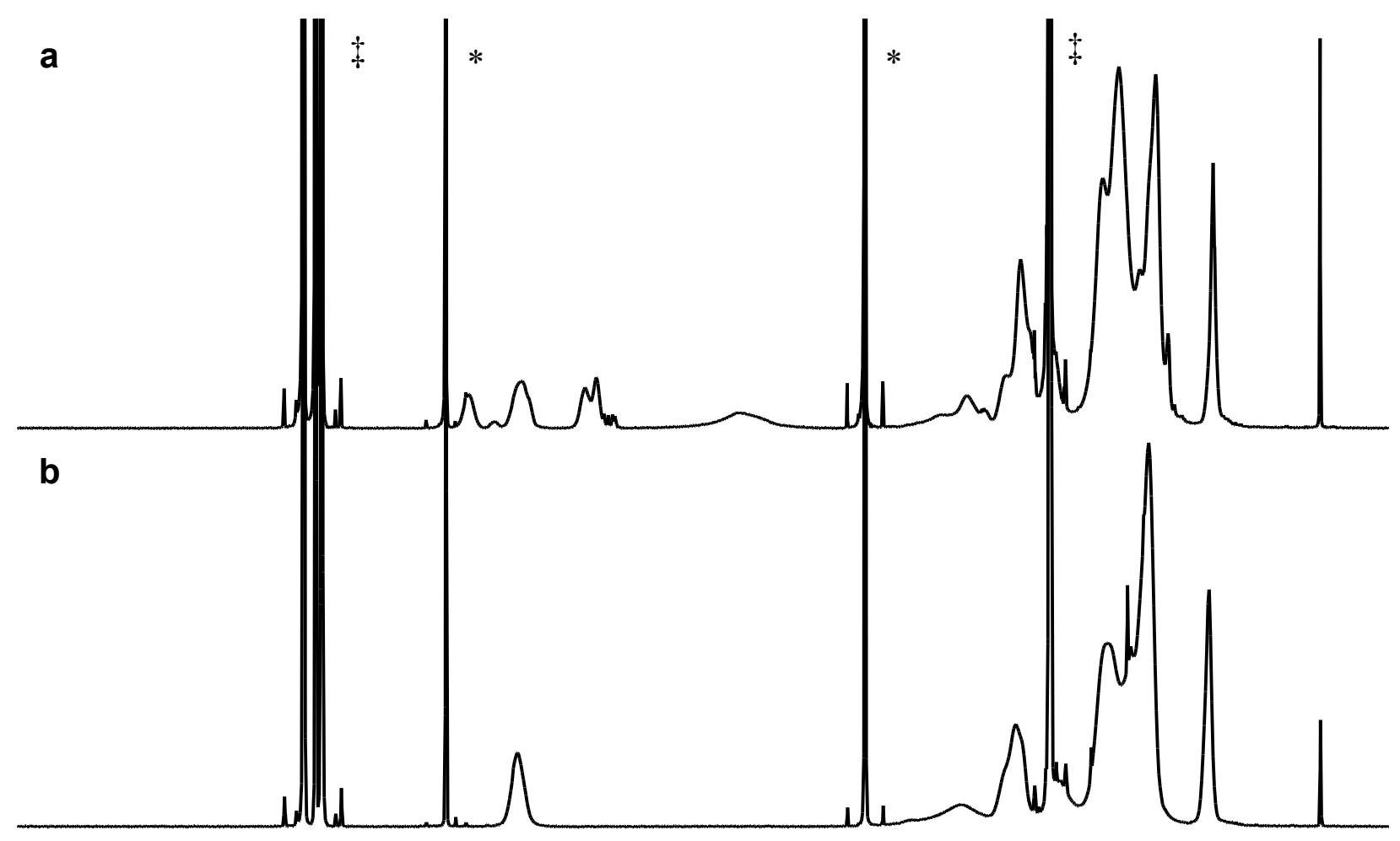

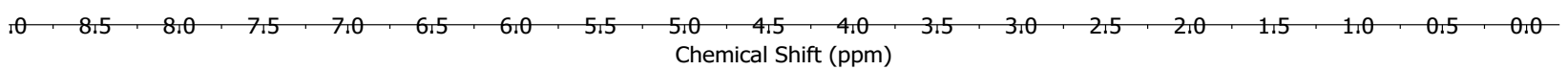

Figure S18. $600 \mathrm{MHz}{ }^{1} \mathrm{H}$ NMR spectra in toluene- $d_{8}$ of a) UDT/oleate-PbS QDs and b) $3.1 \mathrm{~nm}$ oleate-PbS QDs. Sharp peaks denoted by $\$$ are toluene solvent residuals, and peaks marked by * are protons within the internal standard 1,3,5-trimethoxybenzene. As mentioned in the main text, a triplet indicative of disulfide presence can be detected at $2.53 \mathrm{ppm}$ for the protons closest to the $\mathrm{S}-\mathrm{S}$ group. For the purified UDT/oleate-PbS QDs, the triplet integrates to ca. 0.3 disulfide per QD, about $5 \%$ of the total unbound UDT species. 


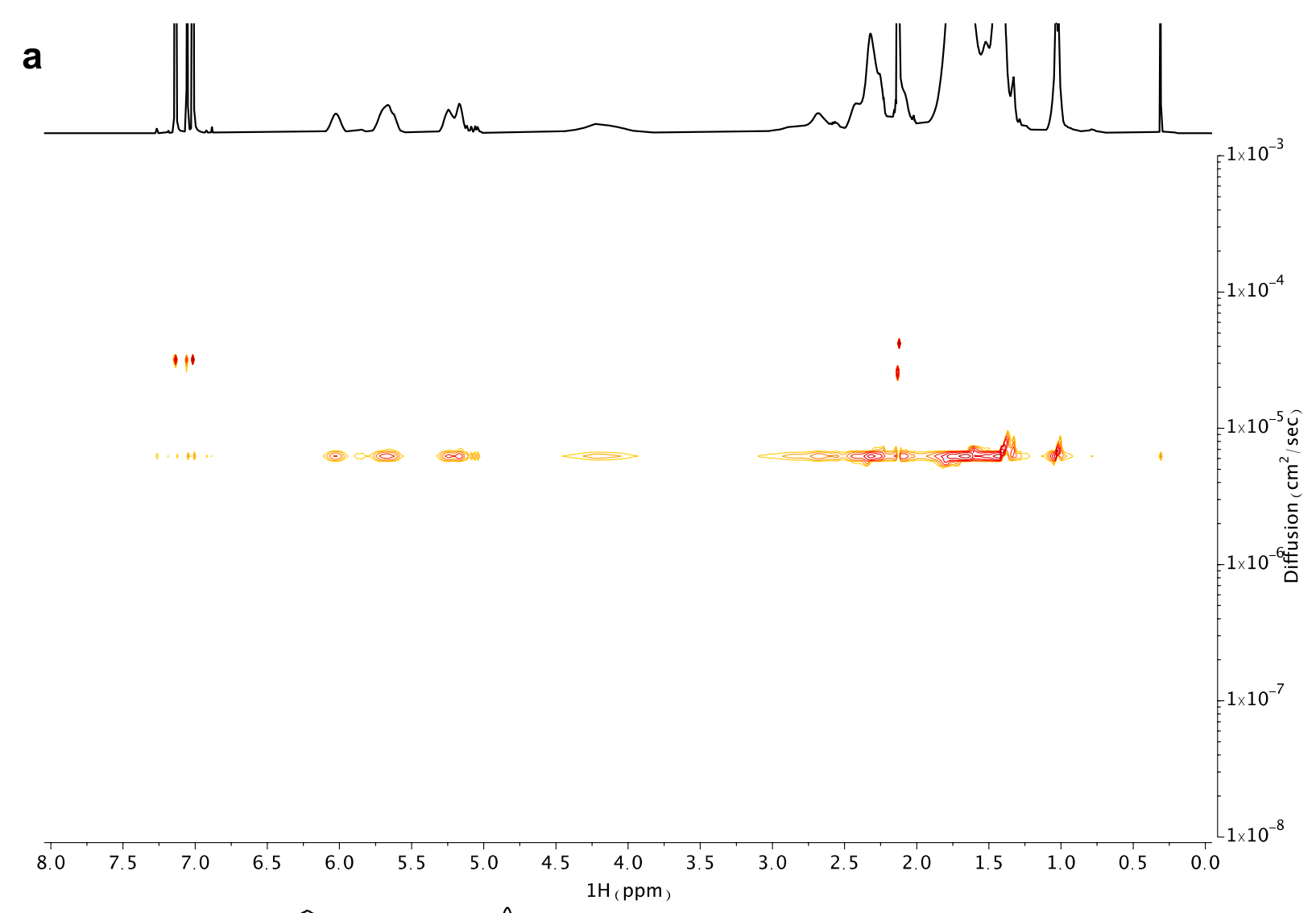

b
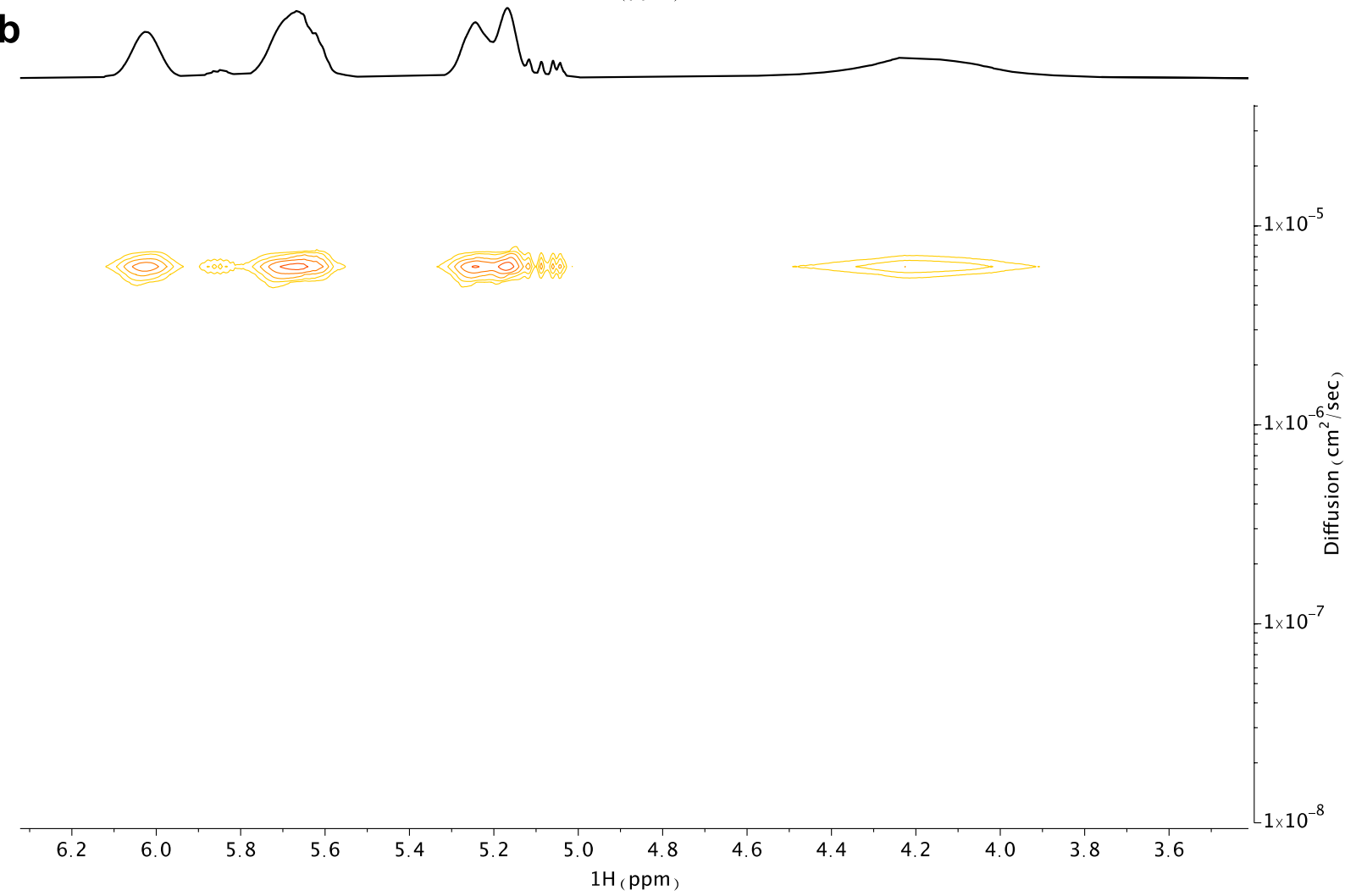

$-1 \times 10^{-5}$ 
Figure S19. a) 2D DOSY plot of $150 \mu \mathrm{M}$ UDT/oleate-PbS QDs in toluene- $d_{8}$. An average $90^{\circ}$ pulse length was determined to be 10 microseconds for the sample, and a delay of 18 seconds was found to be sufficient for relaxation. Spectra were acquired with the stebpg1s pulse sequence to minimize relaxation effects. A delta of $100 \mathrm{~ms}$ and gradient pulse length of 1100 microseconds were used. An array of 16 gradient strengths with a linear sampling was chosen for this experiment. The array of protons was baseline corrected and fit using the three-parameter fitting function in MNOVA, indicating single exponential decay, and a Bayesian transformation (RF Factor: 2, Repetitions: 2) was applied to the array. b) Zoomed-in DOSY plot centered on the alkene region. All "bound" and "free" UDT alkene peaks diffuse together (approx. 6.3 $10^{-}$ ${ }^{6} \mathrm{~cm}^{2} / \mathrm{s}$ ), indicating the "free" thiol species is strongly associated with the quantum dot. Additionally, the small diffusion constant for the ligand resonances indicates that they are bound to the QD surface. 
Ligand displacement and surface charging in oleate-PbS and mixed-shell PbS QDs monitored via ${ }^{1} \mathrm{H}$ NMR and $\mathrm{UV}-\mathrm{V}$ is with excess $\mathrm{CoCp} 2$ added

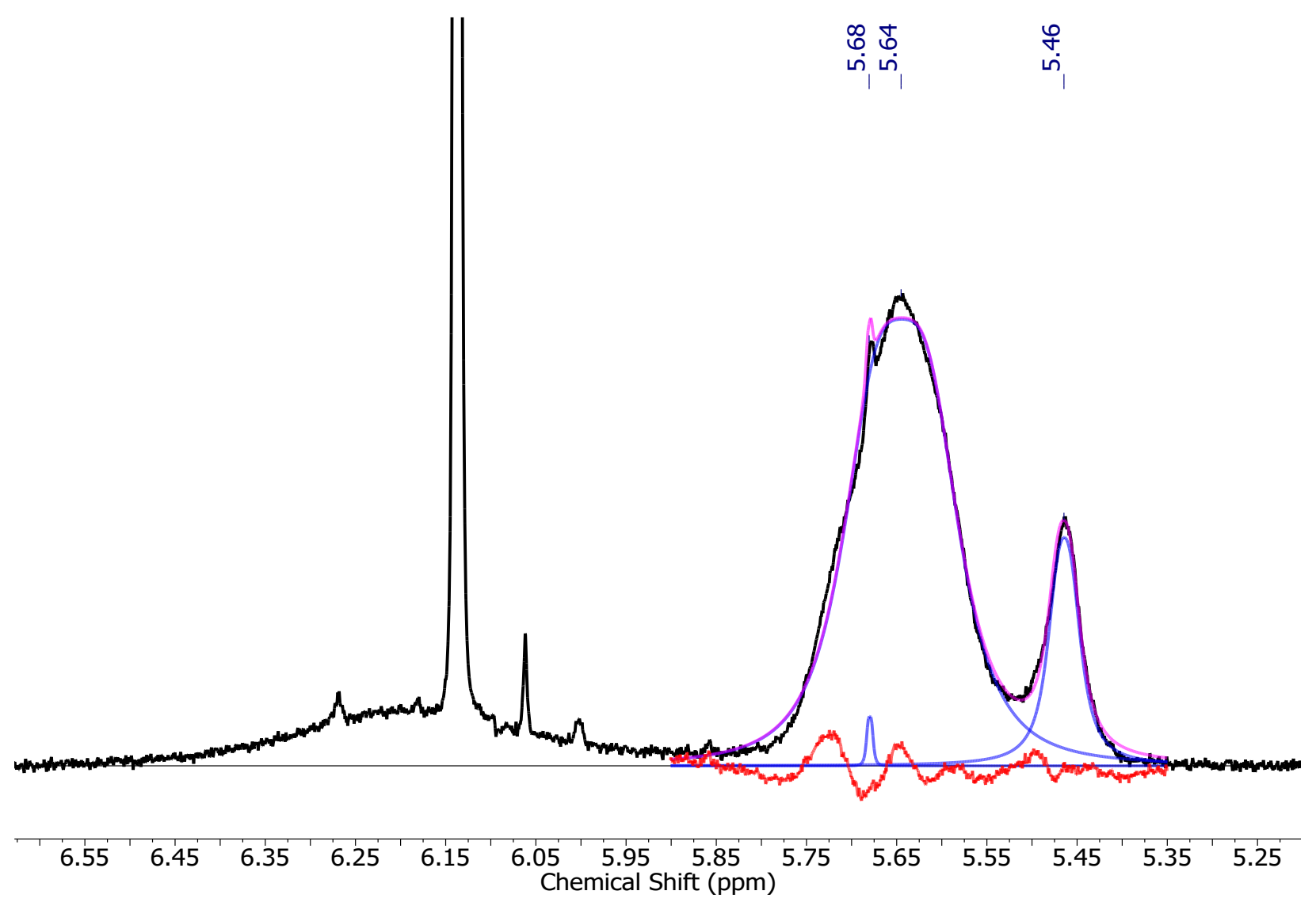

Figure S20. Multipeak fitting functions of the bound and free oleate ligands shown here for oleatePbS-1 QD with 500 eq. CoCp 2 added after 123 hours. The original spectrum is shown in the black trace, the fits are shown as blue traces with the sum shown as a pink trace. Bound oleate was fit to a single peak situated at $5.64 \mathrm{ppm}$. Free oleate was fit to a single peak located at $5.46 \mathrm{ppm}$. The residual of the fit is shown in red. Peak at 5.68 is minor impurity. 


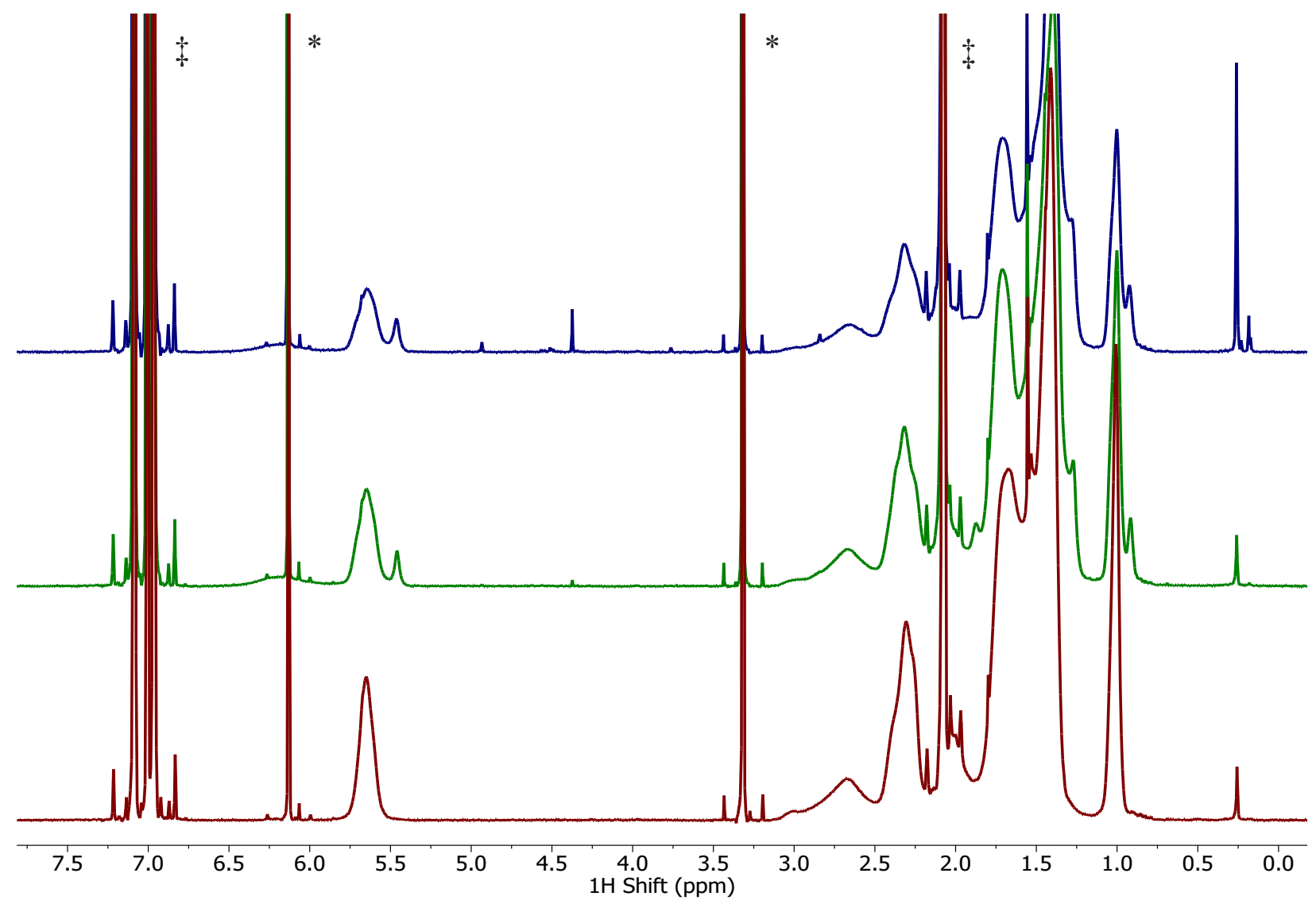

Figure S21. $600 \mathrm{MHz}{ }^{1} \mathrm{H}$ NMR spectra in toluene- $d_{8}$ of oleate-PbS-1 QDs with 0 (red), 100 (green), and 500 (blue) eq CoCp 2 added after 123 hours. Peaks denoted by $\ddagger$ are toluene solvent residuals, and peaks marked by * are the internal standard 1,3,5-trimethoxybenzene. 


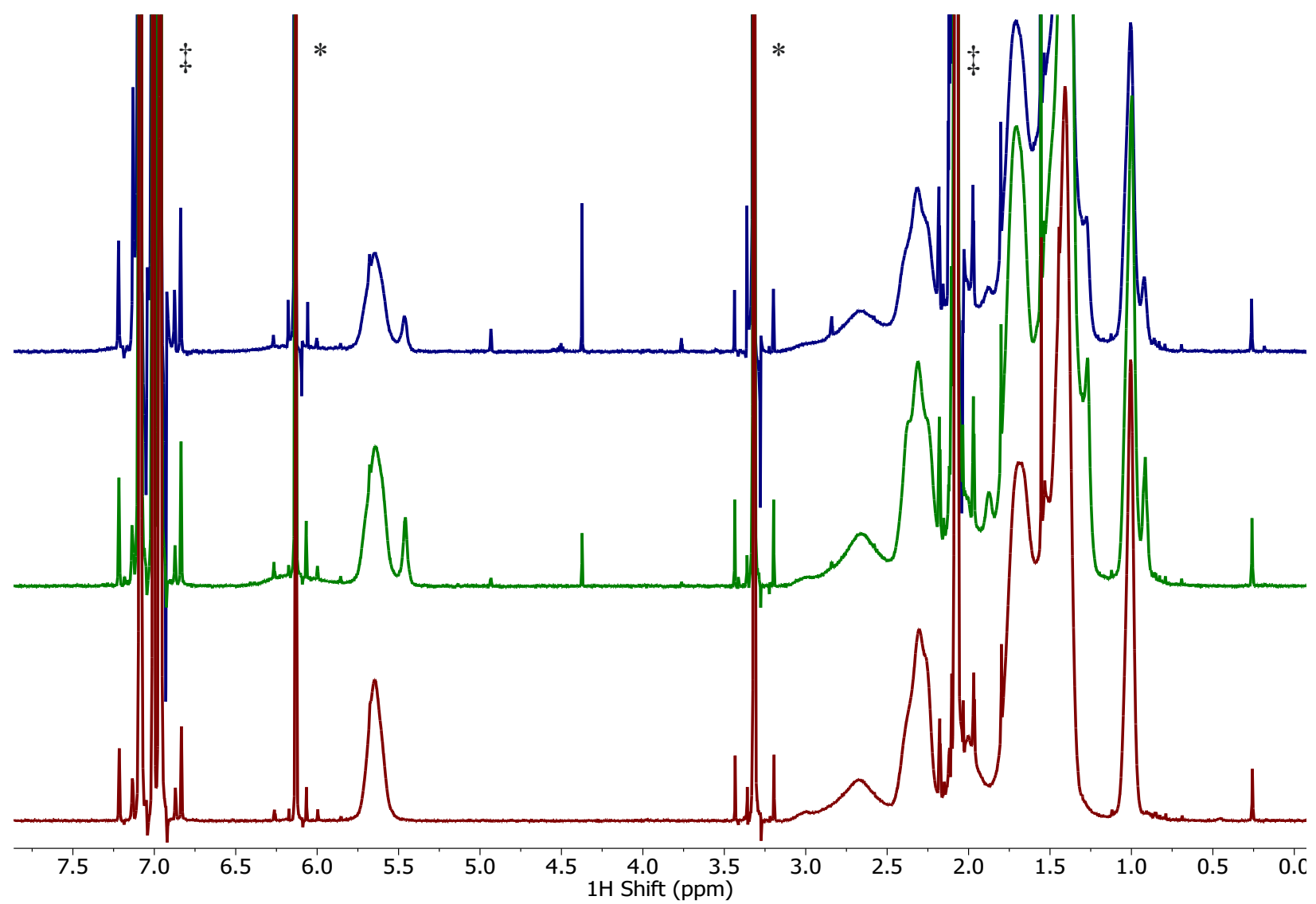

Figure S22. $600 \mathrm{MHz}{ }^{1} \mathrm{H}$ NMR spectra in toluene- $d_{8}$ of oleate-PbS-2 QDs with 0 (red), 100 (green), and 500 (blue) eq CoCp 2 added after 123 hours. Peaks denoted by $\ddagger$ are toluene solvent residuals, and peaks marked by * are the internal standard 1,3,5-trimethoxybenzene. 


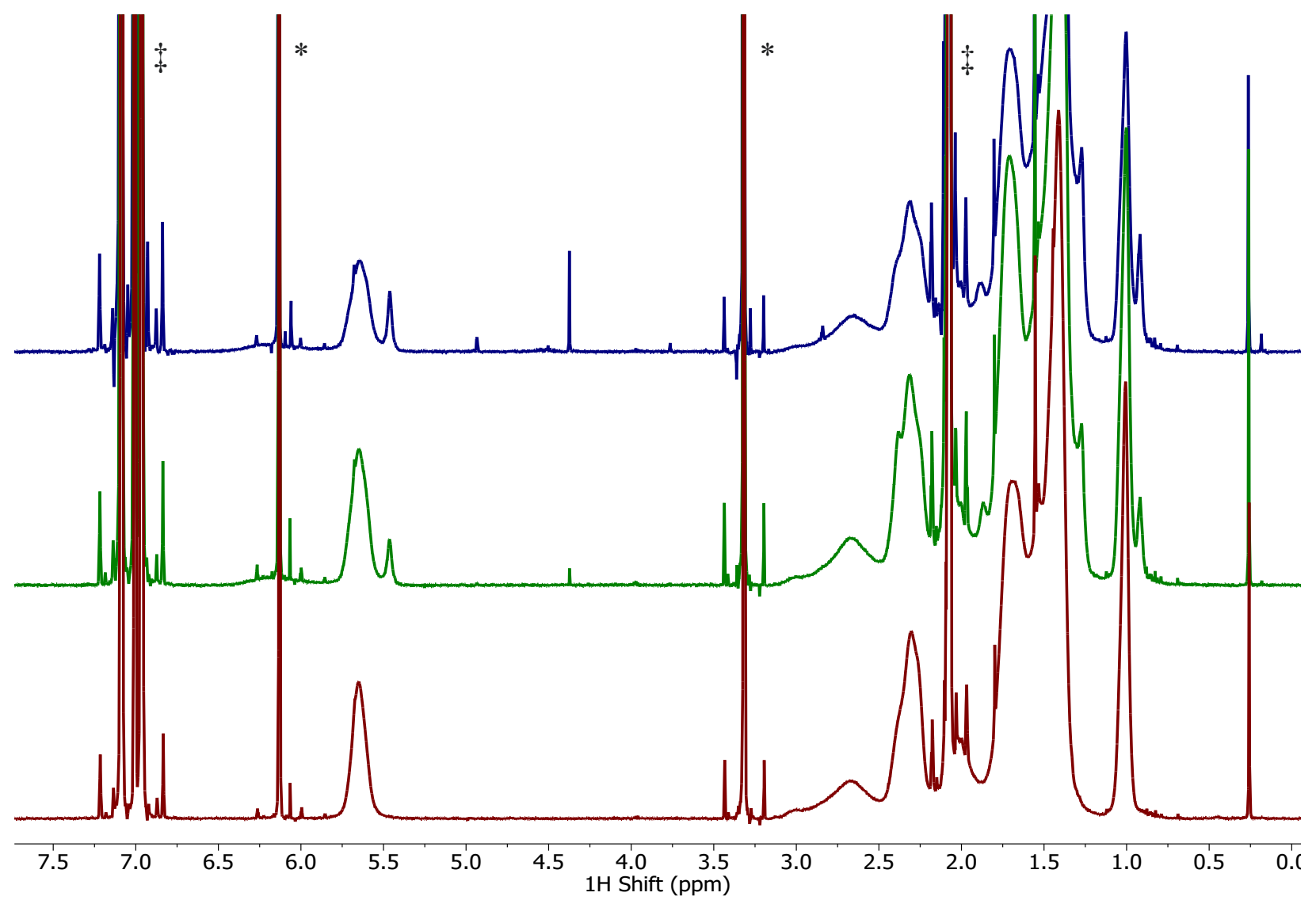

Figure S23. $600 \mathrm{MHz}{ }^{1} \mathrm{H}$ NMR spectra in toluene- $d_{8}$ of oleate-PbS-3 QDs with 0 (red), 100 (green), and 500 (blue) eq CoCp 2 added after 123 hours. Peaks denoted by $\ddagger$ are toluene solvent residuals, and peaks marked by * are the internal standard 1,3,5-trimethoxybenzene. 


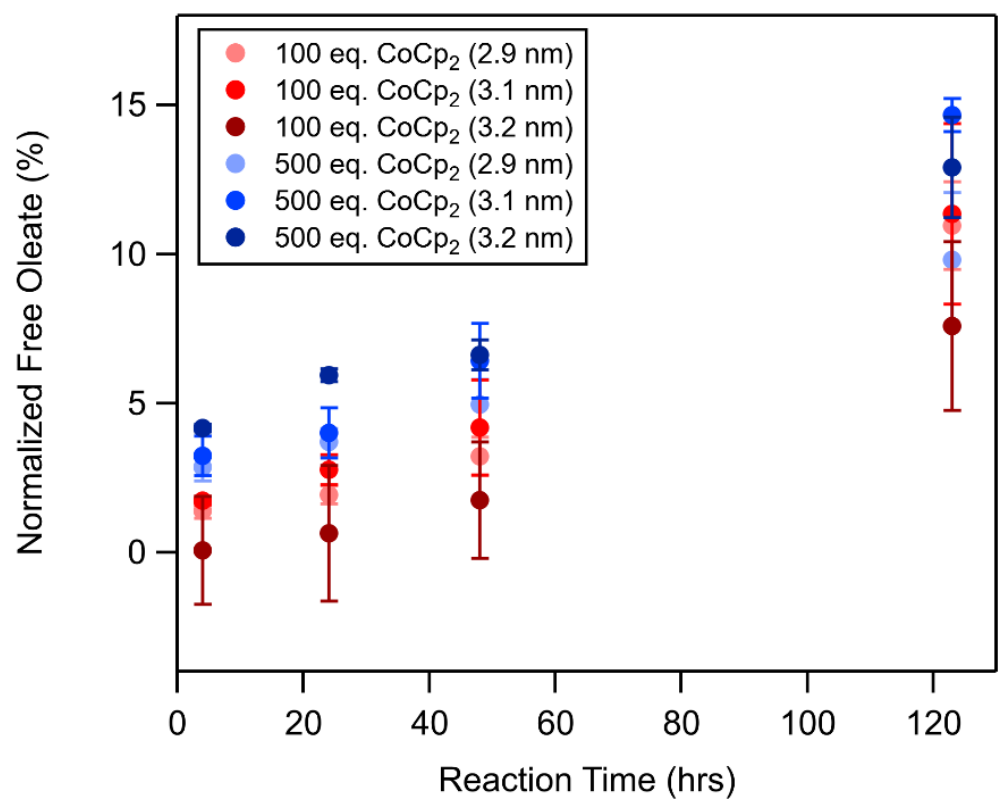

Figure S24. a) Quantification of oleate ligand displaced over time in native oleate-capped QD batches with 100 eq $\mathrm{CoCp}_{2}$ added and b) 500 eq $\mathrm{CoCp}_{2}$ added (average values from triplicate runs \pm standard deviation).

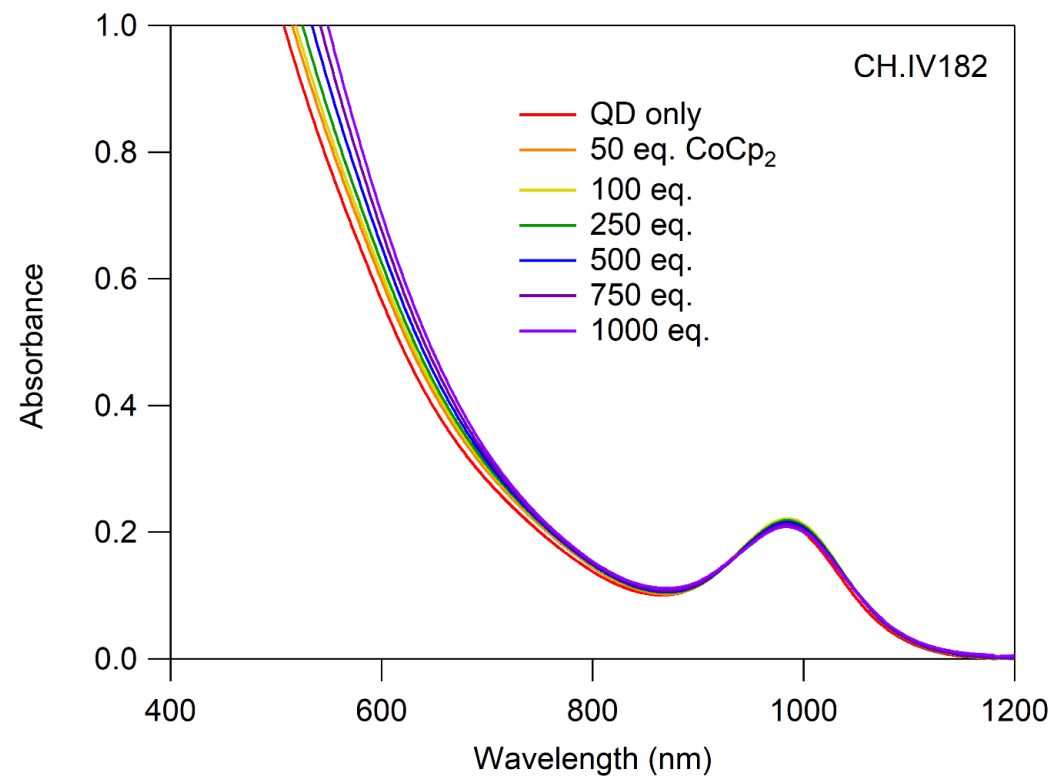

Figure S25. UV-Vis-NIR absorption spectrum of oleate-PbS-1 in toluene $(2.5 \mu \mathrm{M})$ with added excess $\mathrm{CoCp}_{2}$. We observe a $4 \mathrm{~nm}$ red shift with added $\mathrm{CoCp}_{2}$. 


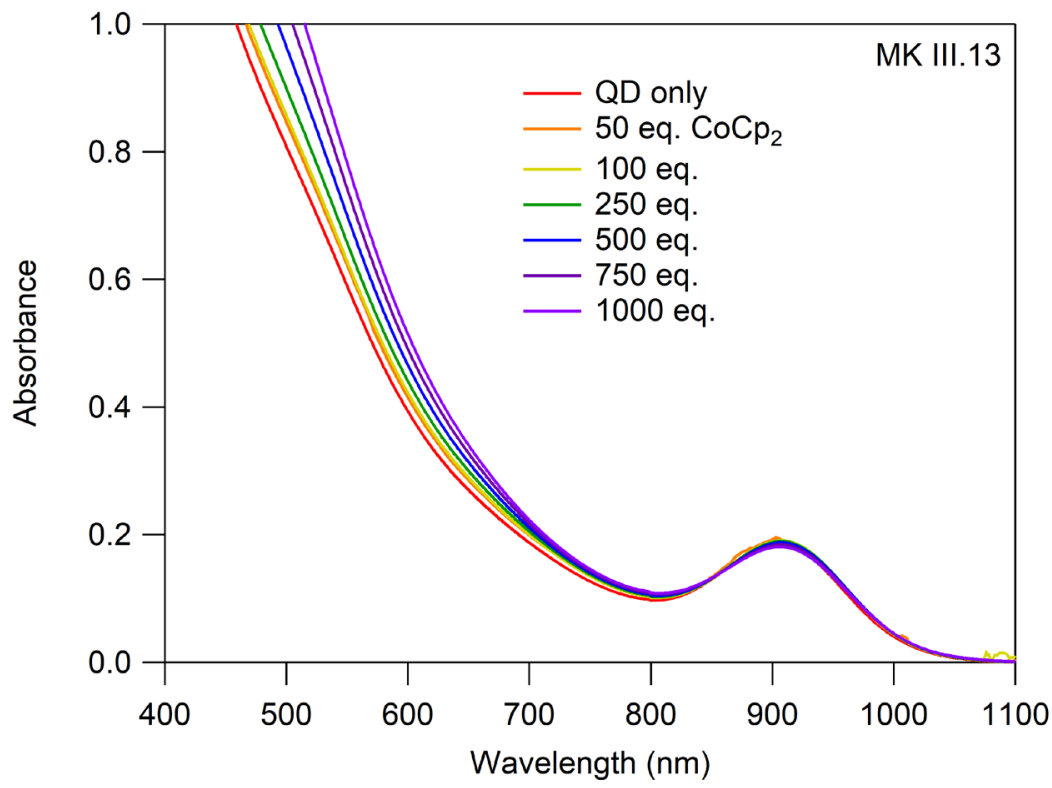

Figure S26. UV-Vis-NIR absorption spectrum of oleate-PbS-2 in toluene $(2.5 \mu \mathrm{M})$ with added excess $\mathrm{CoCp}_{2}$. We observe a $2 \mathrm{~nm}$ red shift with added $\mathrm{CoCp} 2$.

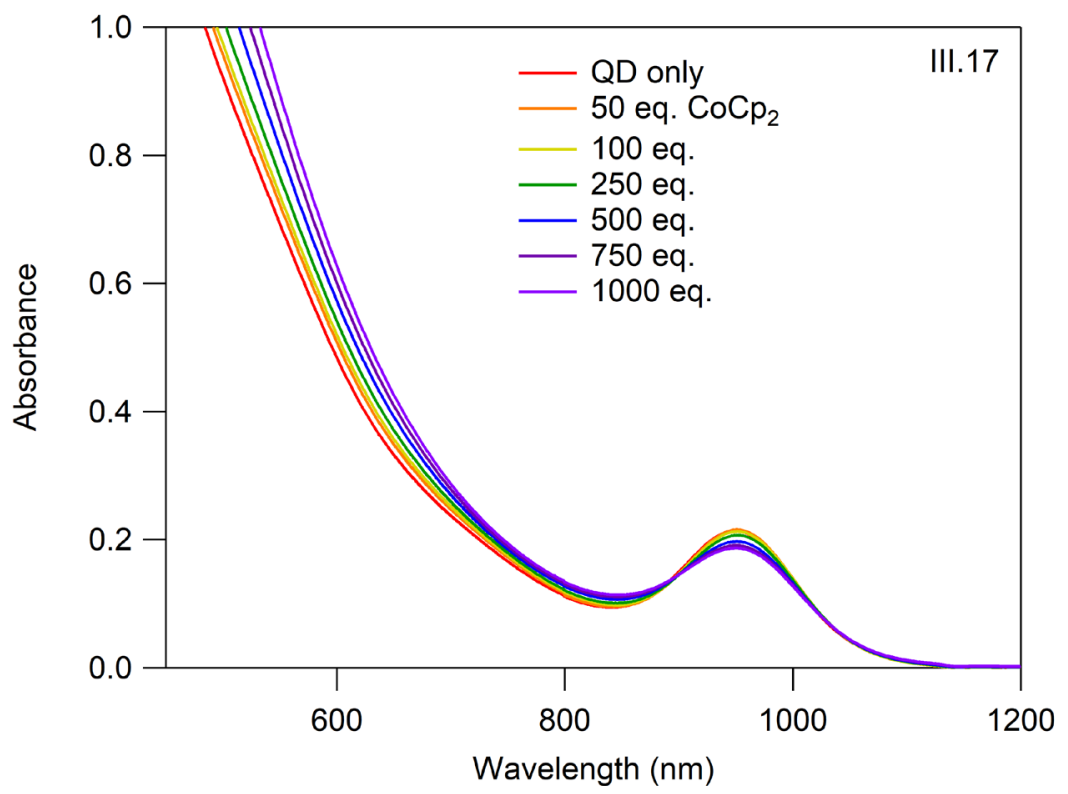

Figure S27. UV-Vis-NIR absorption spectrum of oleate-PbS-3 in toluene $(2.5 \mu \mathrm{M})$ with added excess $\mathrm{CoCp}_{2}$. We observe a $2 \mathrm{~nm}$ red shift and minor loss of absorbance with added CoCp 2 . 


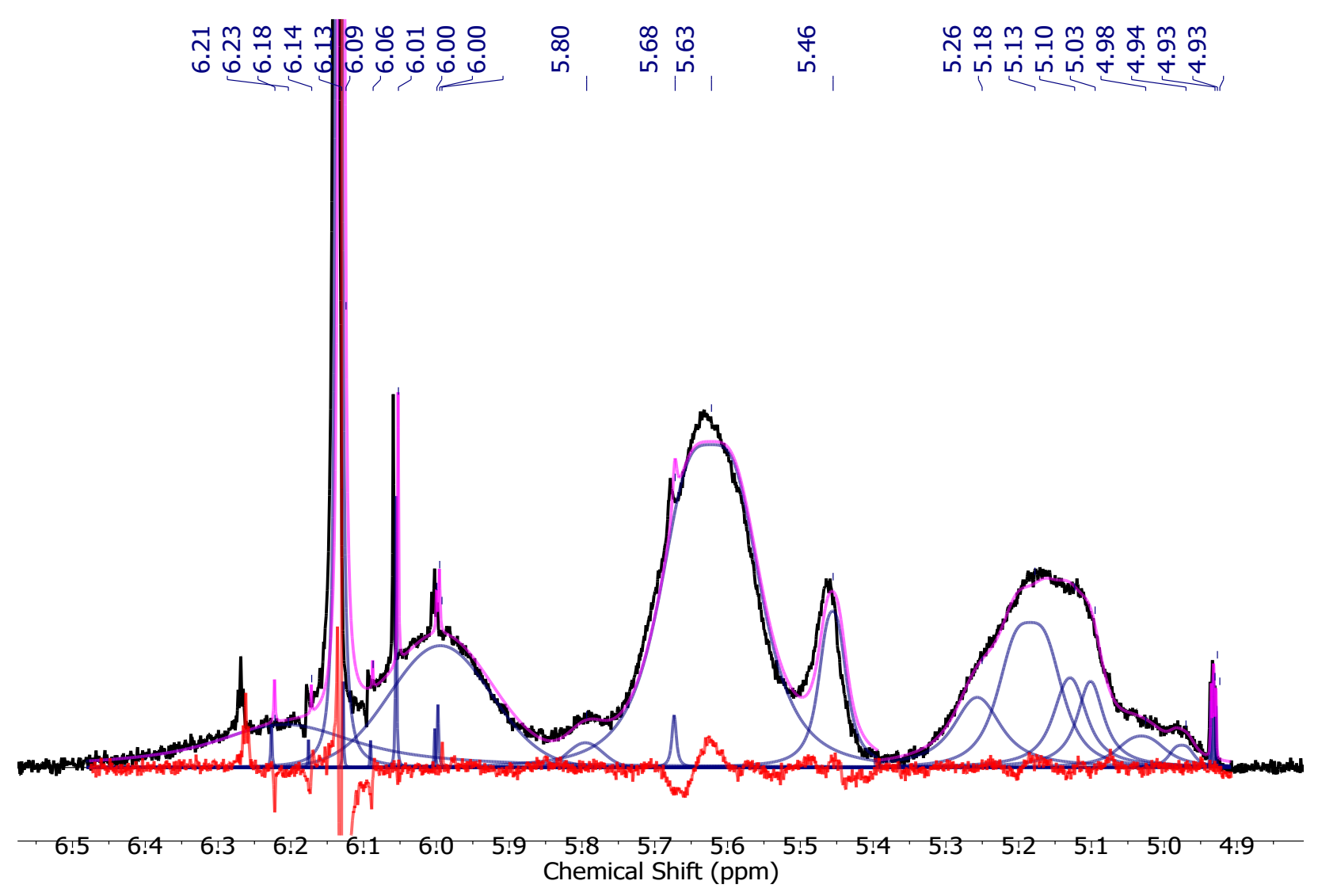

Figure S28. Multipeak fitting functions of the bound and free ligands shown here for UDA/oleate$\mathrm{PbS}$ QD with 500 eq. CoCp 2 added after 123 hours. The original spectrum is shown in the black trace, the fits are shown as blue traces with the sum shown as a pink trace. Bound oleate was fit to a single peak located at $5.63 \mathrm{ppm}$ and free oleate was fit to a single peak at $5.46 \mathrm{ppm}$. Bound UDA was calculated by averaging the broad peak at $6.00 \mathrm{ppm}$ (alkene $\mathrm{CH}, 1 \mathrm{H}$ ) and the four peaks between 5.26 and $5.10 \mathrm{ppm}$ (alkene $\mathrm{CH}_{2}, 2 \mathrm{H}$ ), normalized to the same number of protons. Free UDA was calculated by averaging the broad peak at $5.80 \mathrm{ppm}(1 \mathrm{H})$ and the two peaks at 5.03 and $4.98 \mathrm{ppm}(2 \mathrm{H})$, normalized to the same number of protons. The residual of the fit is shown in red. Peaks at $5.68 \mathrm{ppm}$ and the triplet at $4.93 \mathrm{ppm}$ are minor impurities. 


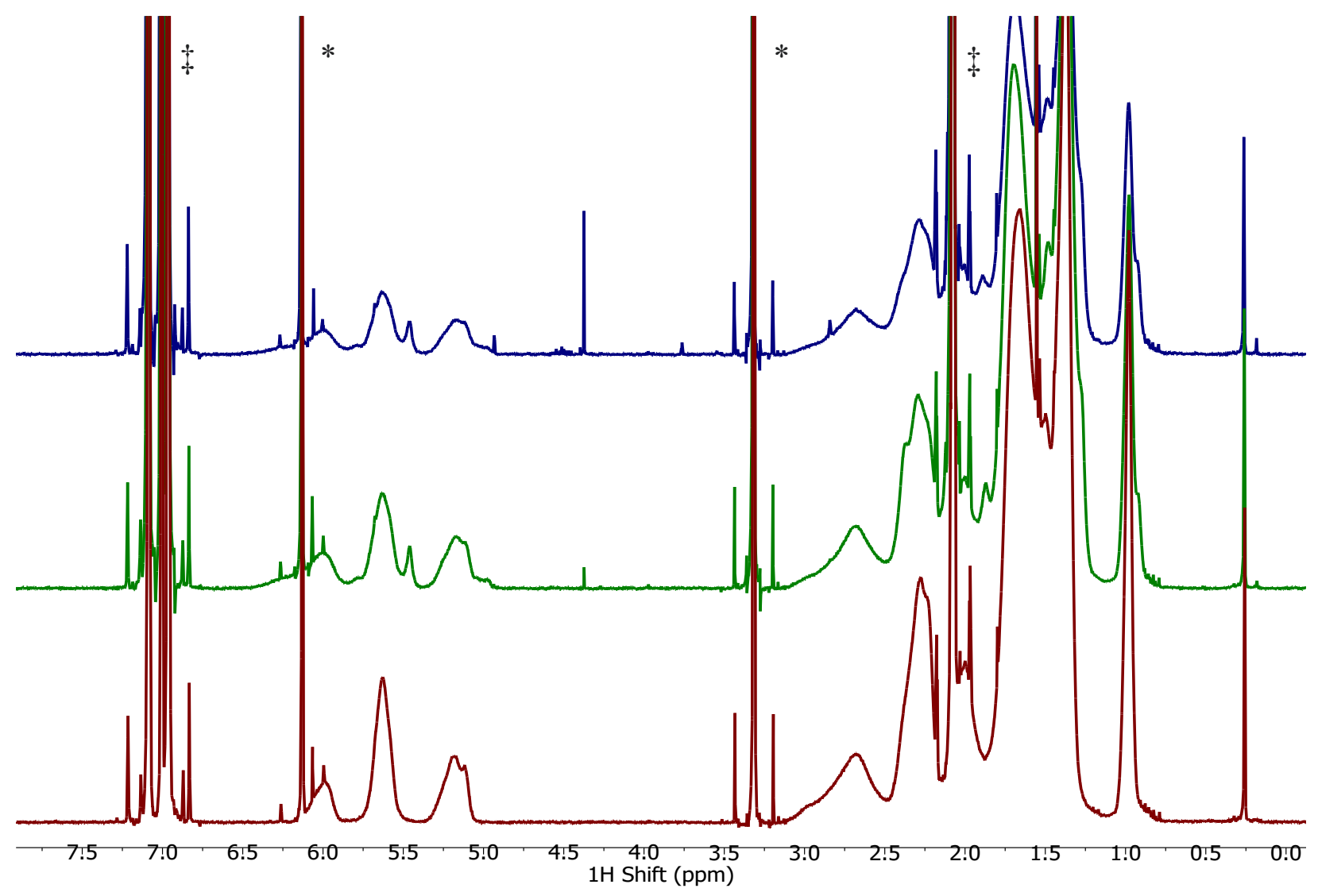

Figure S29. $600 \mathrm{MHz}{ }^{1} \mathrm{H}$ NMR spectra in toluene- $d_{8}$ of UDA/oleate-PbS QDs with 0 (red), 100 (green), and 500 (blue) eq CoCp 2 added after 123 hours. Peaks denoted by $\ddagger$ are toluene solvent residuals, and peaks marked by * are the internal standard 1,3,5-trimethoxybenzene. 


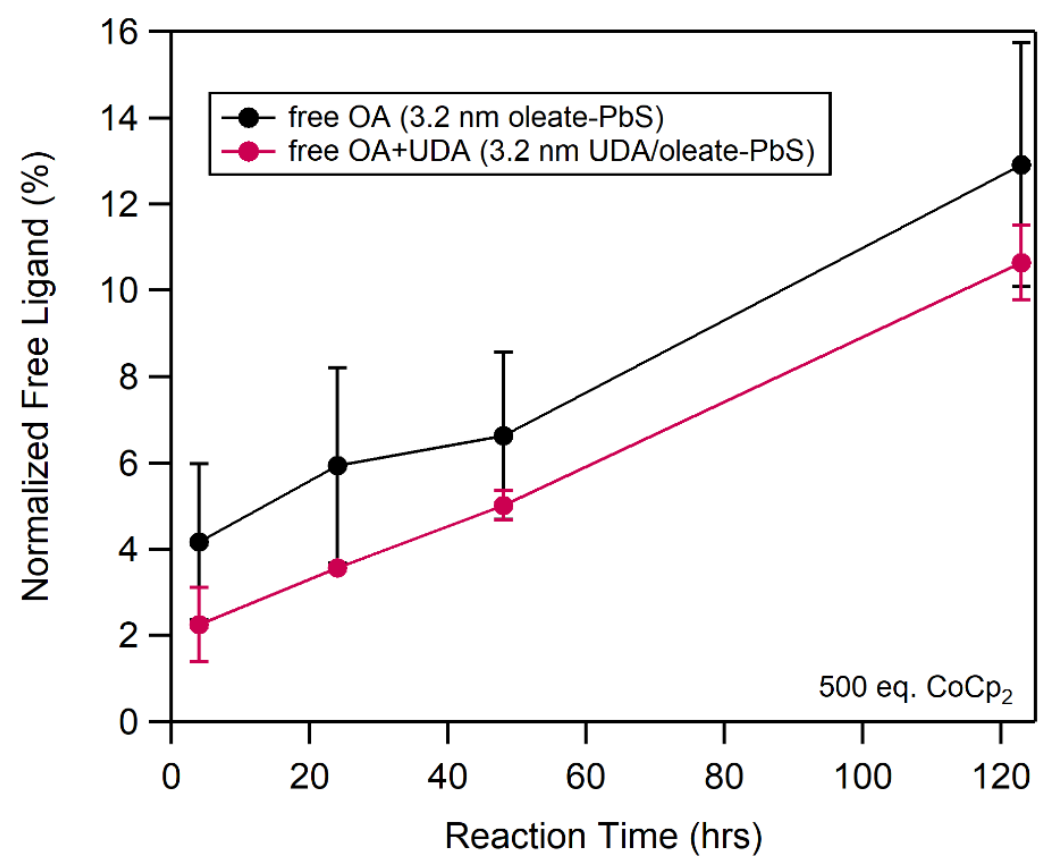

Figure S30. Quantification of ligand displaced in oleate-PbS-1 (black) and UDA/oleate-PbS (pink) batches with 500 eq $\mathrm{CoCp}_{2}$ added over time (average values from triplicate runs \pm standard deviation). Lines connecting data points are a guide to the eye.

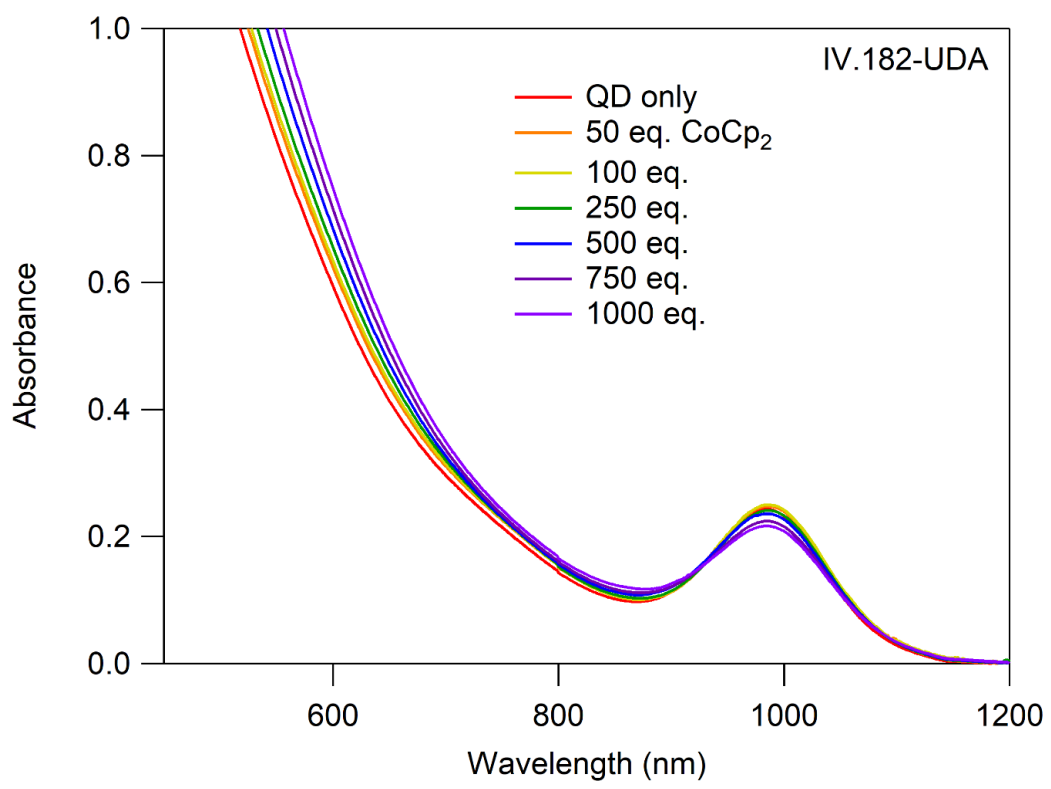

Figure S31. UV-Vis-NIR absorption spectrum of UDA/oleate-PbS in toluene $(2.5 \mu \mathrm{M})$ with added excess $\mathrm{CoCp}_{2}$. We observe a $2 \mathrm{~nm}$ red shift and minor loss of absorbance with added $\mathrm{CoCp}_{2}$. 


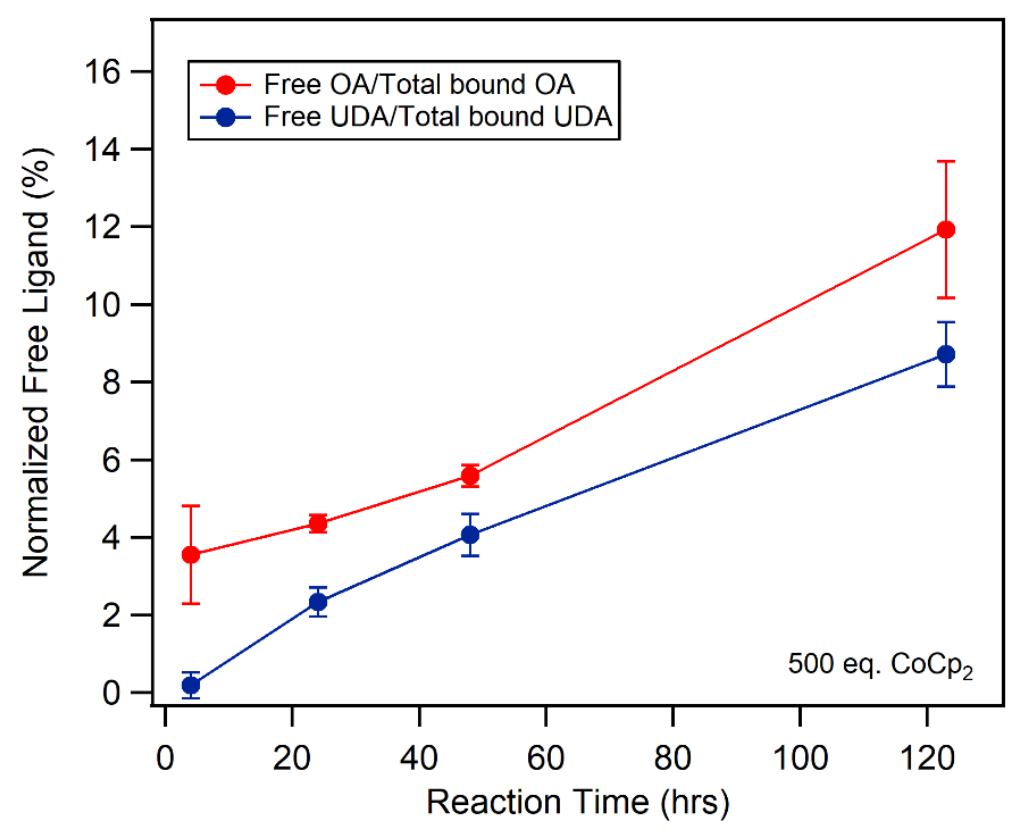

Figure S32. Quantification of the amount of displaced oleate (red, normalized to the starting amount of bound oleate) versus displaced UDA (blue, normalized to the starting amount of bound $\mathrm{UDA}$ ) in UDA/oleate- $\mathrm{PbS}$ with $500 \mathrm{eq} \mathrm{CoCp}_{2}$ added over time (average values from triplicate runs \pm standard deviation). Lines connecting data points are a guide to the eye. 


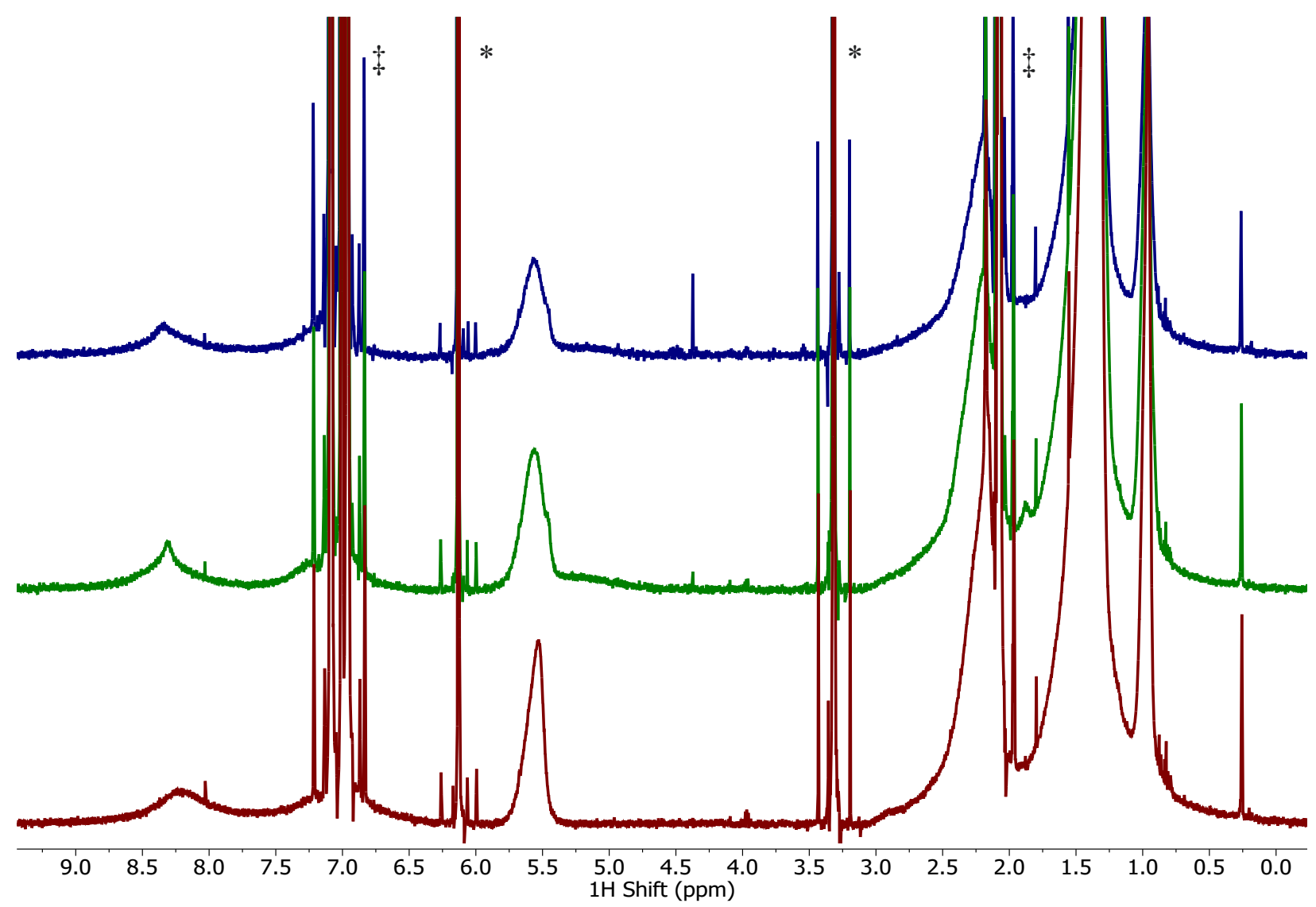

Figure S33. $600 \mathrm{MHz}{ }^{1} \mathrm{H}$ NMR spectra in toluene- $d_{8}$ of toluate/oleate-PbS QDs with 0 (red), 100 (green), and 500 (blue) eq CoCp 2 added after 123 hours. Peaks denoted by $\ddagger$ are toluene solvent residuals, and peaks marked by * are the internal standard 1,3,5-trimethoxybenzene. 


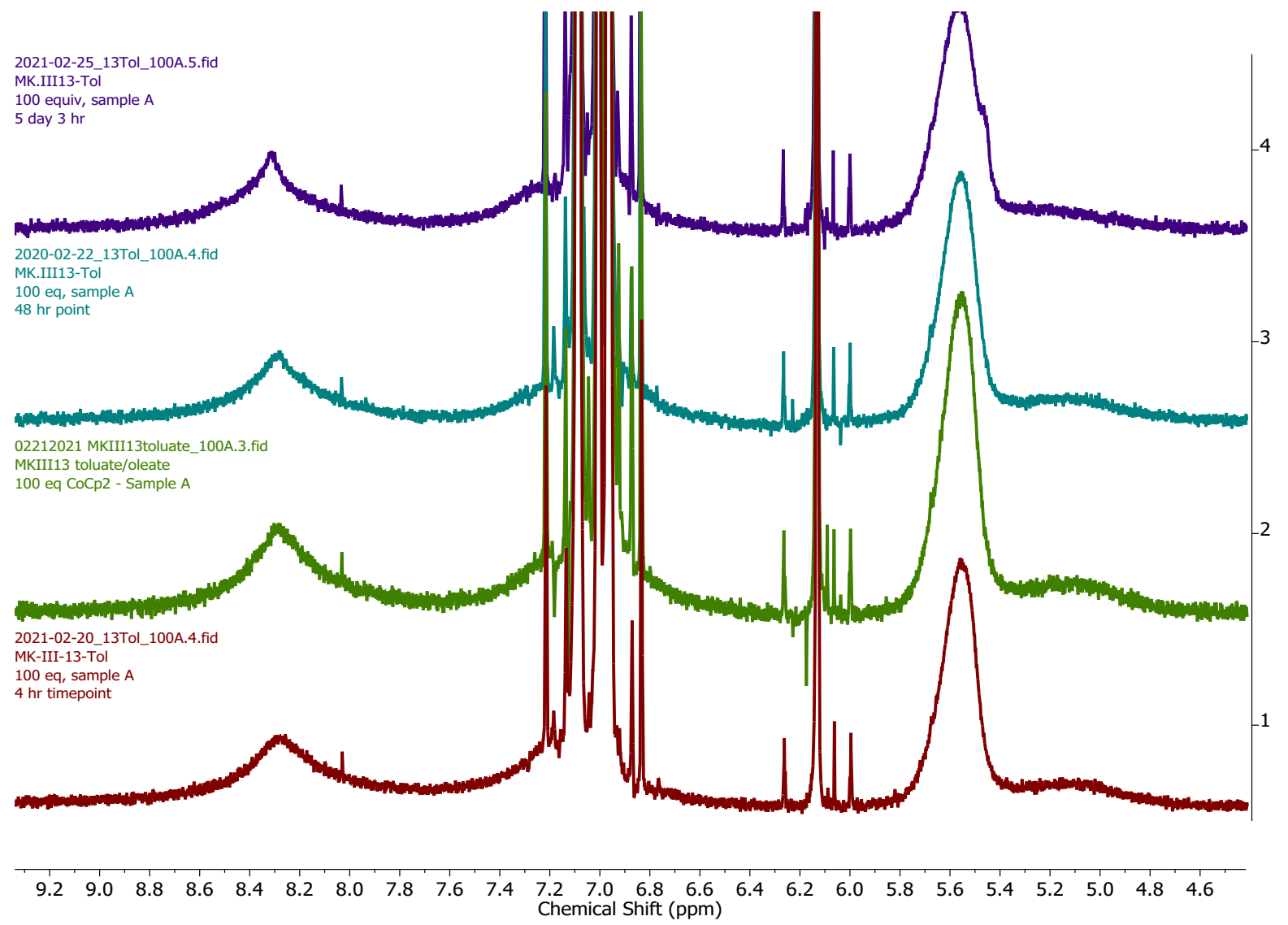

Figure S34. Toluate/oleate-PbS QDs in toluene- $d_{8}$ with 100 eq. CoCp 2 added at 4 hours (red), 24 hours (green), 48 hours (blue) and 123 hours (purple). Over time the sharp free toluate peak at 8.3 ppm shifts downfield. 


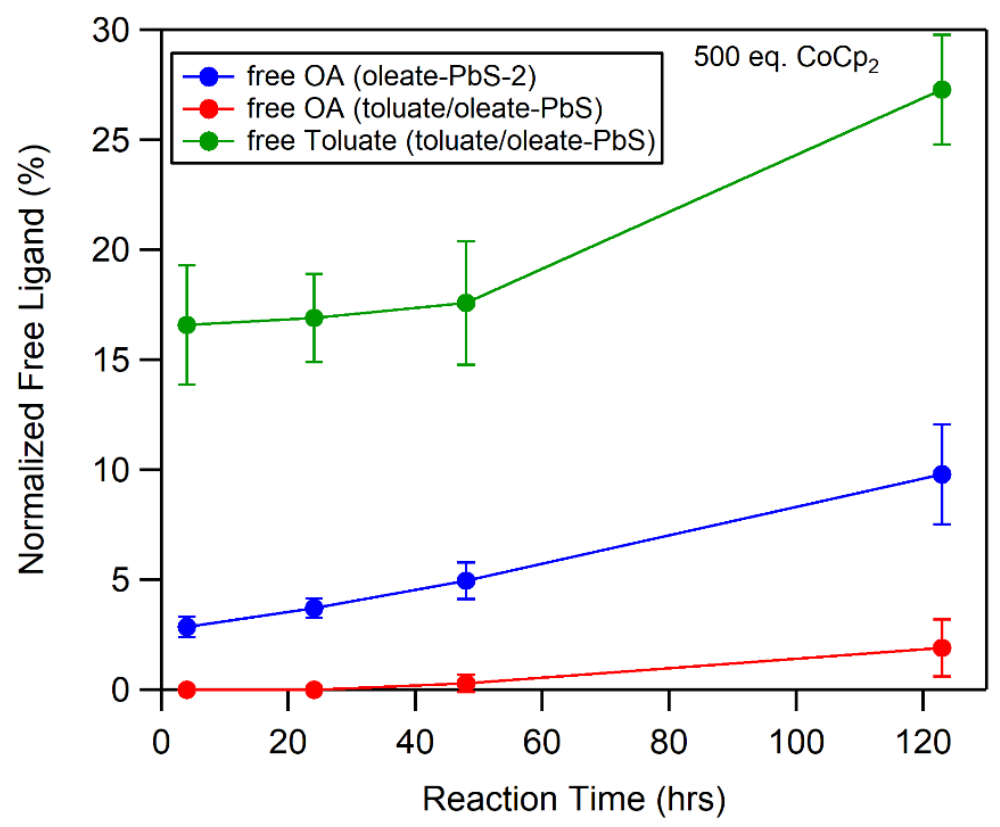

Figure S35. Quantification of free ligand in oleate-PbS-2 (blue) and free toluate (green) and free oleate (red) in toluate/oleate- $\mathrm{PbS}$ batches over time with 500 eq $\mathrm{CoCp}_{2}$ added (average values from triplicate runs \pm standard deviation). Lines connecting data points are a guide to the eye.

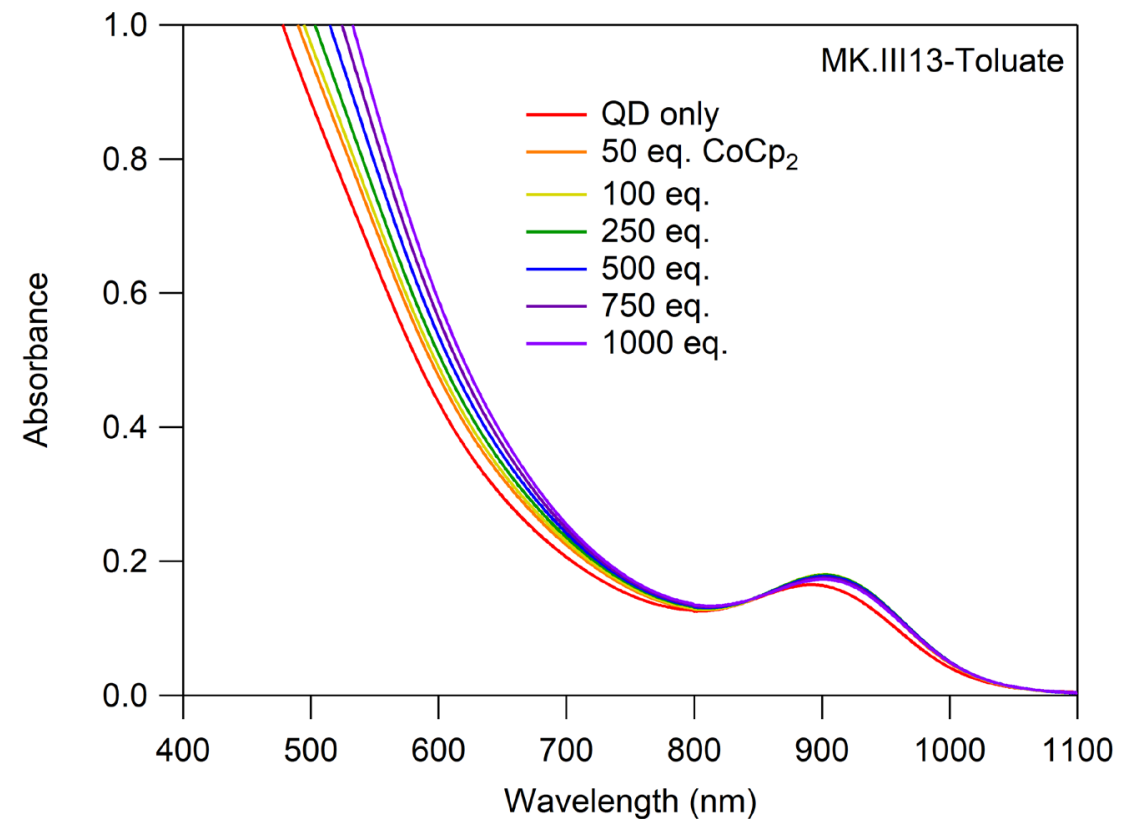

Figure S36. UV-Vis-NIR absorption spectrum of toluate/oleate-PbS in toluene $(2.5 \mu \mathrm{M})$ with added excess $\mathrm{CoCp}_{2}$. We observe a $10 \mathrm{~nm}$ red shift with added $\mathrm{CoCp}_{2}$. 


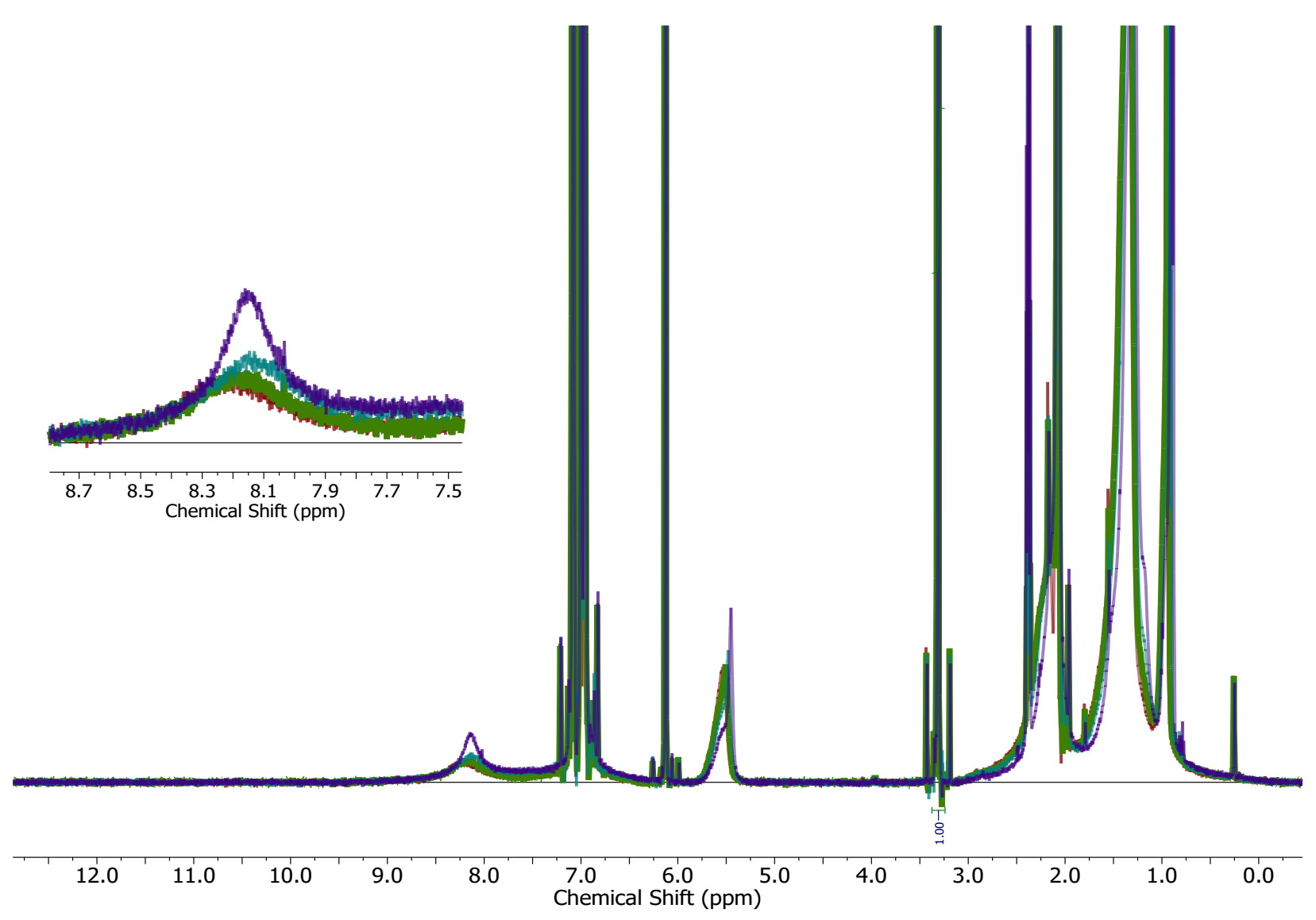

Figure S37. Toluate/oleate- $\mathrm{PbS}$ in toluene- $d_{8}$ with added $\mathrm{TEAH}^{+}$-Toluate ligand showing sharpening and shift up field with added free ligand: 0 eq. TEAH ${ }^{+}$-Toluate (green), 5 eq. TEAH ${ }^{+}$Toluate (red), 15 eq. TEAH+-Toluate (blue), 40 eq. TEAH ${ }^{+}$-Toluate (purple). Inset: Zoom-in of toluate ligand peak.

Due to the overlapping nature of both the bound and free toluate peaks as well as the oleate and $\mathrm{CoCp}_{2}^{+}$peaks, a strategy different from the other systems was used to obtain consistent integrations for each bound and free ligand species. First, a multipoint baseline correction was applied to each toluate-containing spectrum studied herein, taking care to avoid over-baselining the toluate and $\mathrm{CoCp}_{2}{ }_{2}$ features. The region between 9.5 and 6.4 did not contain any points, nor did the region between 6.0 and $4.7 \mathrm{ppm}$. Next, the bound and free toluate resonances near $8.3 \mathrm{ppm}$ were deconvoluted by fixing the bound feature to $8.2 \mathrm{ppm}$ and allowing a sharp, downfield peak to float. Qualitatively, upon fitting multiple spectra between 4 and 123 hours of reaction, we observed that increasing the area attributed to the free toluate species over time described the data well. However, we recognize that choices in baseline selection and peak position locking may not accurately represent the true chemical nature of the bound and free toluate species. We thus ascribe an error of $30 \%$ of the value of free toluate species liberated $(27 \%)$, which is $8.1 \%$. We attribute $20 \%$ of this error to the fitting procedure, and $10 \%$ to the standard deviation between samples. To further verify that fitting the toluate feature to bound and free species can be justified, we carried out chemical separation methods to analyze the supernatant of this reaction (Figures S38-42). 


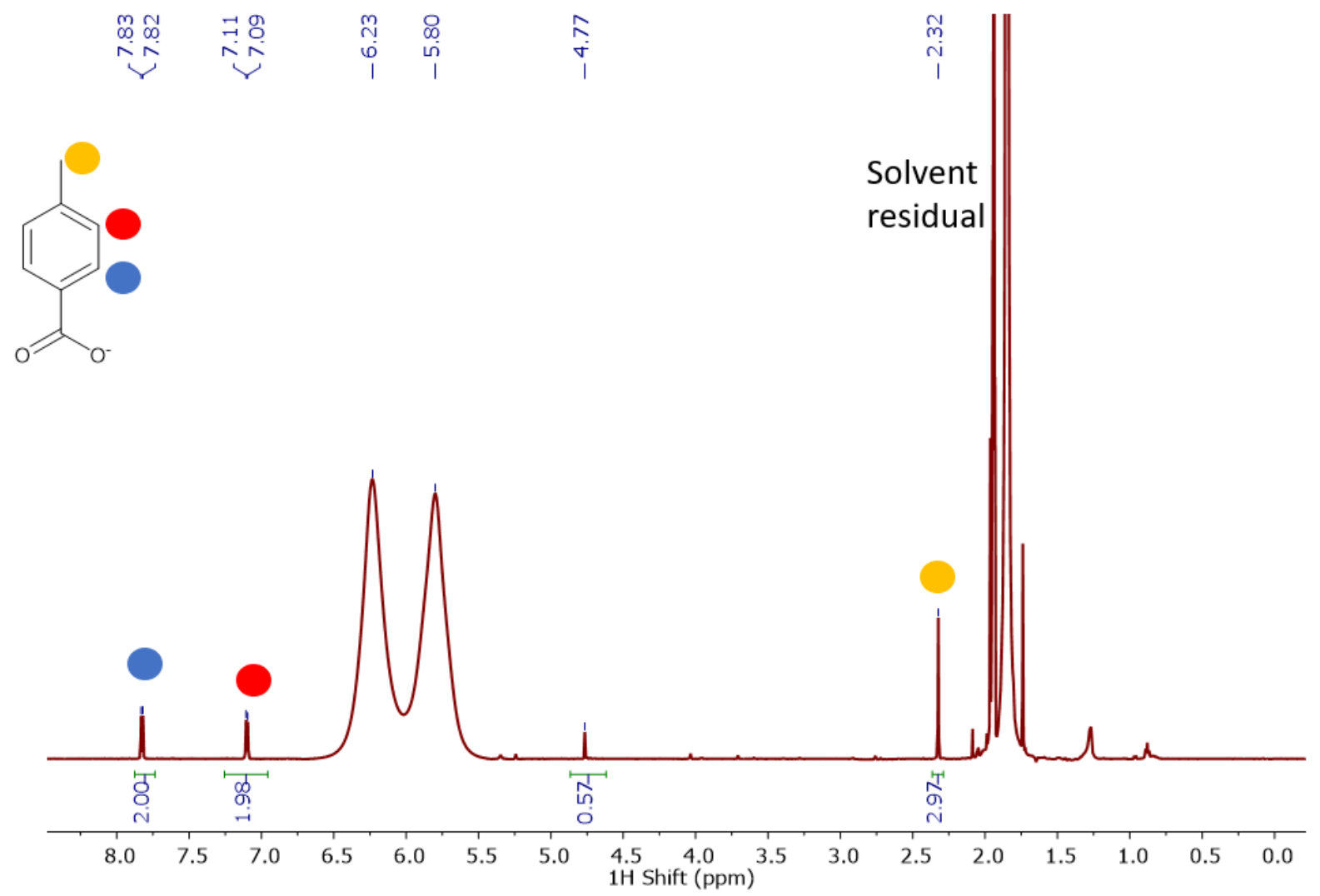

Figure S38. ${ }^{1} \mathrm{H}$ NMR of supernatant from isolating free toluate ligand from reduced QDs through precipitation in acetonitrile- $d_{3}$. Paramagnetic cobaltocene was detected at ca. $-44 \mathrm{ppm}$. The peaks attributed to free toluate compare well with that of $\left[\mathrm{TEAH}^{+}\right][$toluate] (Figure S42). 


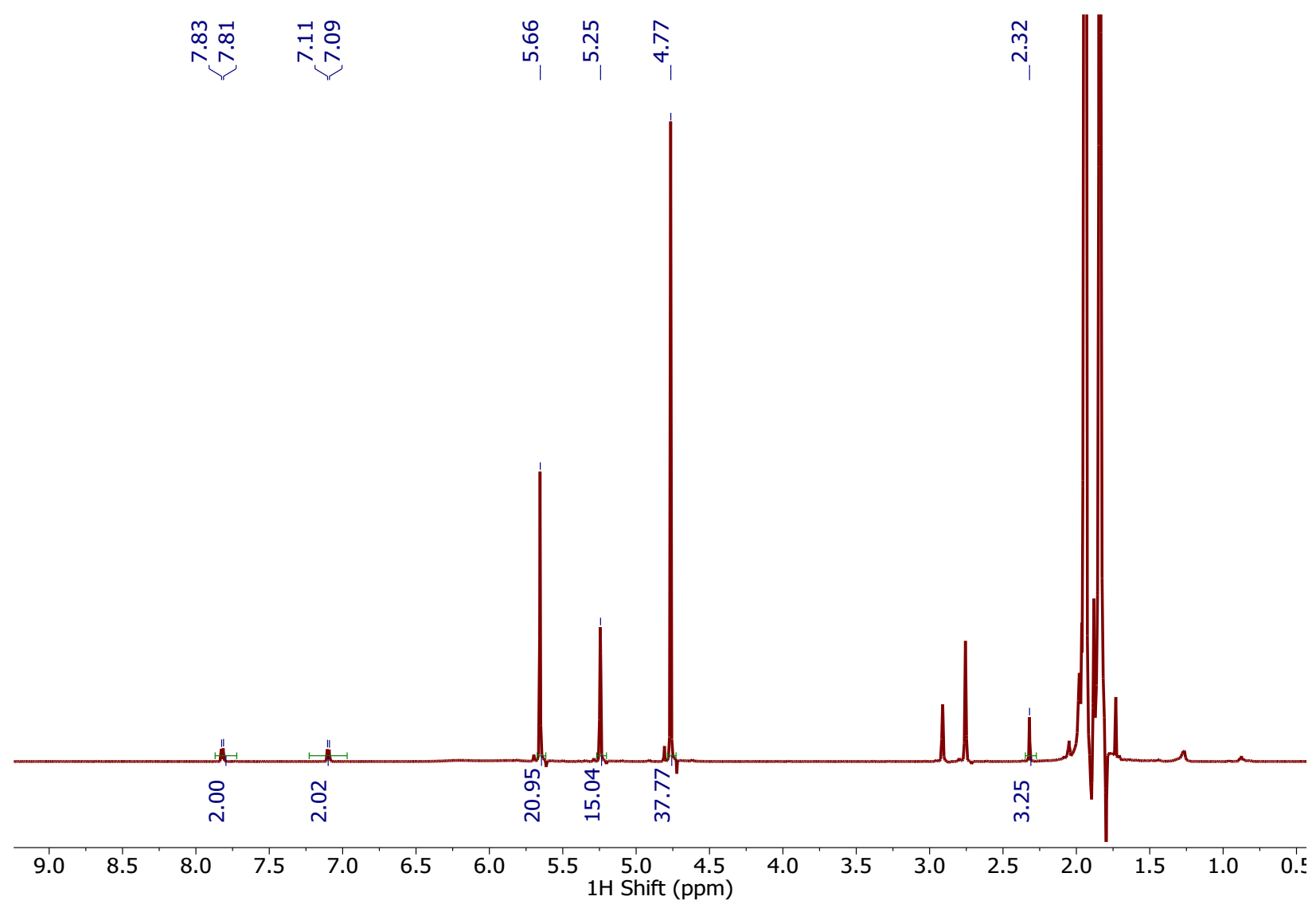

Figure S39. ${ }^{1} \mathrm{H}$ NMR of supernatant from isolating free toluate ligand from reduced QDs through precipitation in acetonitrile- $d_{3}$ upon oxidation with air, showing the sharpening of the $\mathrm{CoCp}_{2}{ }^{+}$ resonance present in excess due to oxidation of paramagnetic $\mathrm{CoCp}_{2}$ in the sample, and paramagnetic impurities. 


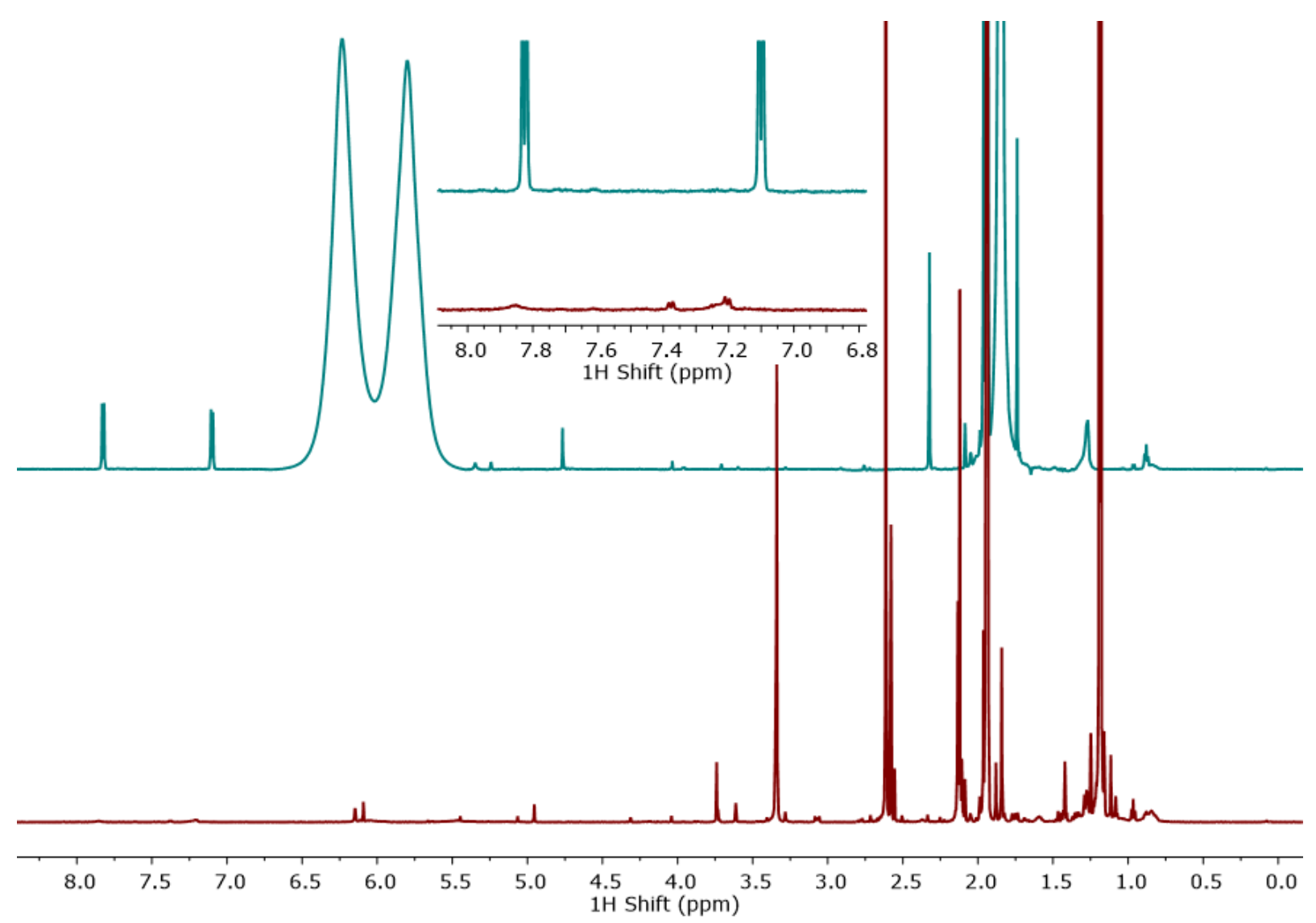

Figure S40. Stacked ${ }^{1} \mathrm{H}$ NMR spectra of (bottom) supernatant from precipitation of toluate/oleate$\mathrm{PbS}$ QDs with acetonitrile and acetone antisolvents, and (top) supernatant from precipitation of toluate/oleate- $\mathrm{PbS}$ reacted with $\mathrm{CoCp}_{2}$. Both NMR samples are in acetonitrile-- $d_{3}$ and were run on a $600 \mathrm{MHz}$ NMR spectrometer. Inset shows a zoom-in of the aryl region showing no free toluate ligand present in the control sample. 


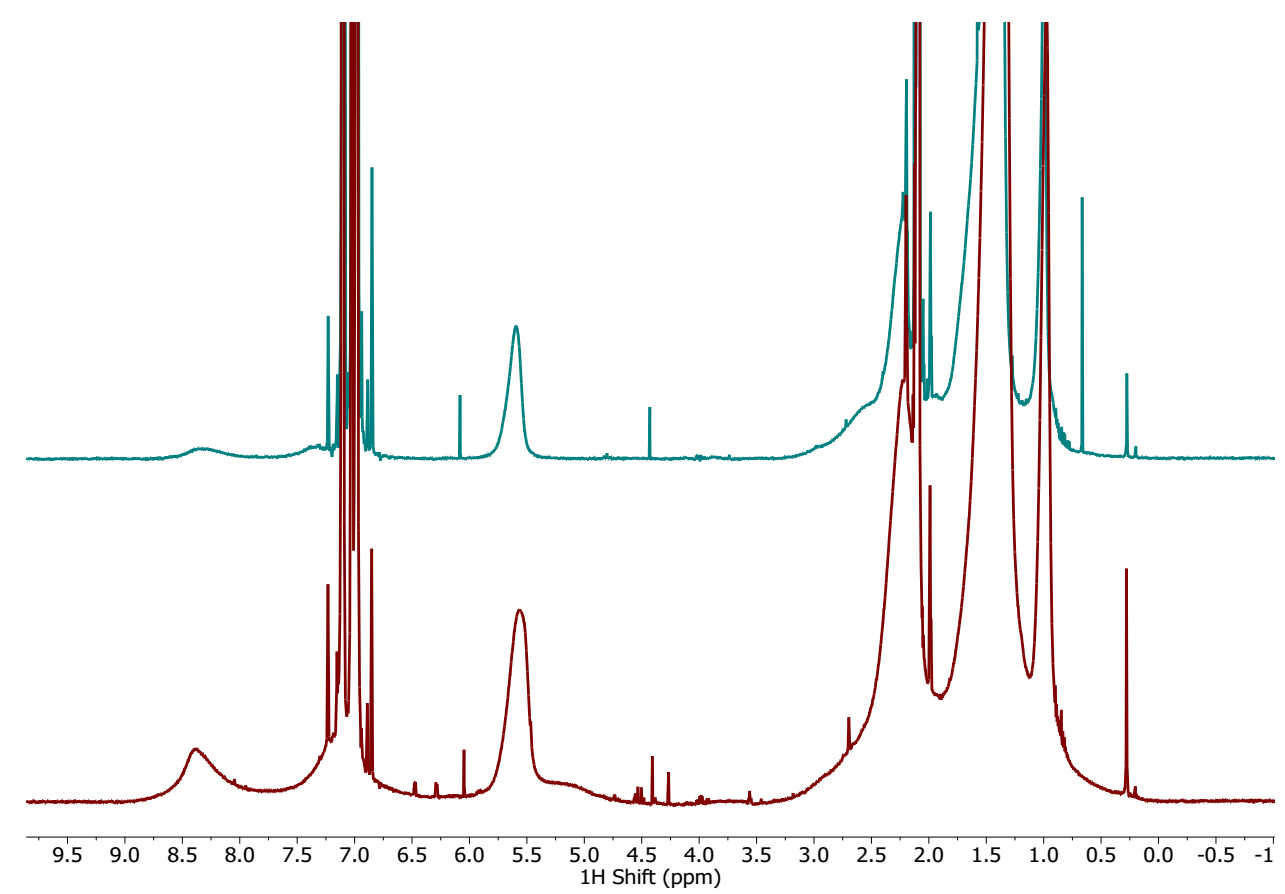

Figure S41. ${ }^{1} \mathrm{H}$ NMR of QD mixture before (bottom) and after (top) precipitation in toluene- $d_{8}$.

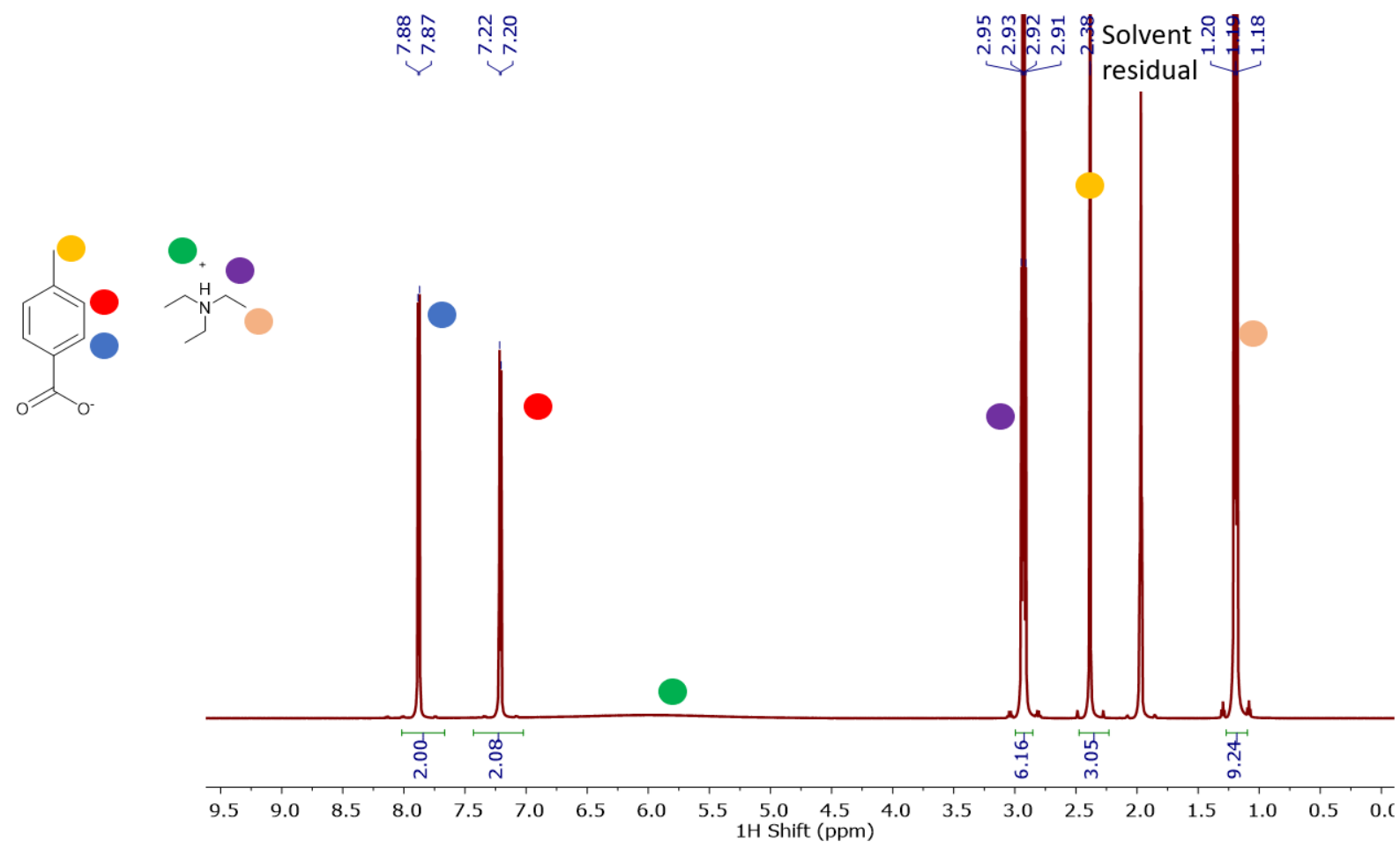

Figure S42. ${ }^{1} \mathrm{H}$ NMR of triethylammonium $p$-toluate in acetonitrile- $d_{3}$. 


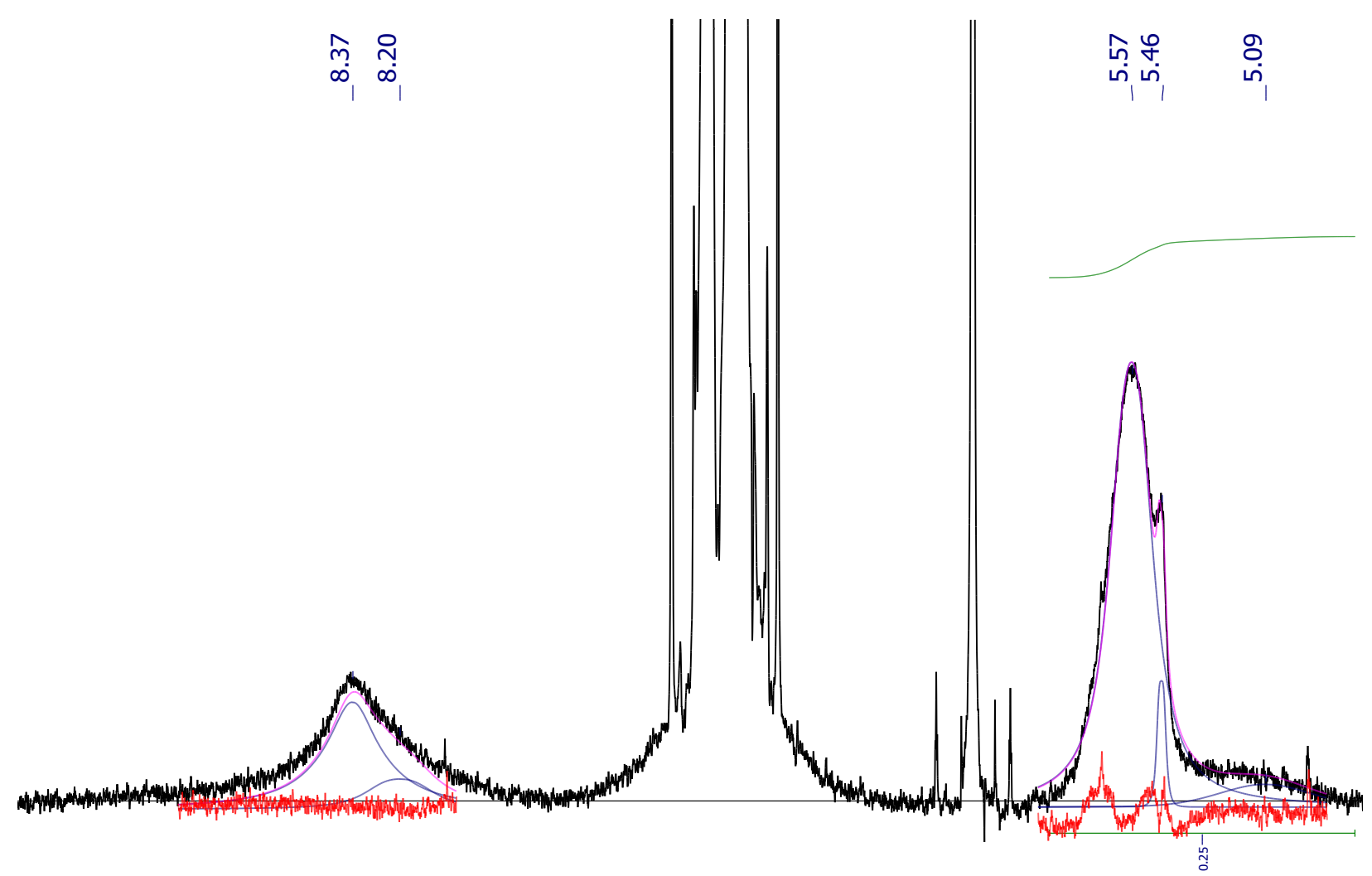

$\begin{array}{lllllllllllllllllllllllllllll}9.4 & 9.2 & 9.0 & 8.8 & 8.6 & 8.4 & 8.2 & 8.0 & 7.8 & 7.6 & 7.4 & 7.2 & 7.0 & 6.8 & 6.6 & 6.4 & 6.2 & 6.0 & 5.8 & 5.6 & 5.4 & 5.2 & 5.0 & 4.8\end{array}$ Chemical Shift (ppm)

Figure S43. Multipeak fitting functions of the bound and free ligands shown here for toluate/oleate-PbS QD with 500 eq. $\mathrm{CoCp}_{2}$ added after 123 hours. The original spectrum is shown in the black trace, the fits are shown as blue traces with the sum shown as a pink trace. The residual of the fit is shown in red. Bound oleate was calculated through subtraction of the broad $\mathrm{CoCp}_{2}^{+}$ resonance at $5.1 \mathrm{ppm}$ and the sharp free oleate resonance at $5.46 \mathrm{ppm}$ from the total integration of the region between 5.9 and $4.8 \mathrm{ppm}$ (shown by green bar). Due to the asymmetry of the bound oleate peak, we found this method gave the most consistent total oleate integration over time and across samples. Bound toluate was fixed $8.2 \mathrm{ppm}$, consistent with the peak shift of toluate/oleate$\mathrm{PbS}$ QDs in the absence of $\mathrm{CoCp}_{2}$. The free toluate feature was allowed to float, and was fit to a sharper feature at $8.37 \mathrm{ppm}$. While subjectivity may be inherent to multipeak NMR fitting of the overlapping toluate aryl resonances, within two extreme bounds of fitting we estimate an error of $5 \%$ in our reported fits. Within the range of $20-30 \%$, however, the toluate/oleate-PbS QDs lose measurably more bound ligand than the other systems explored herein. 


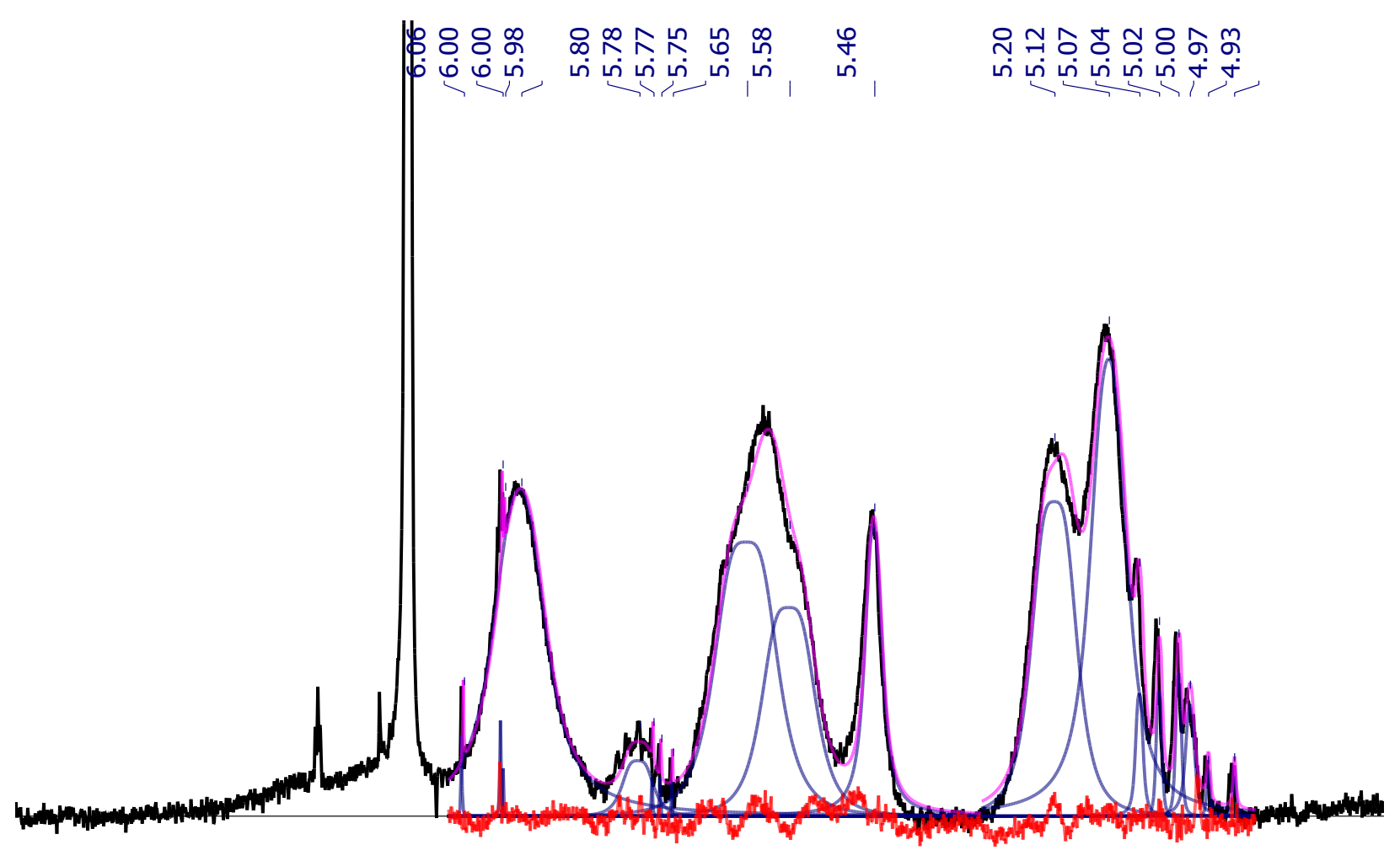

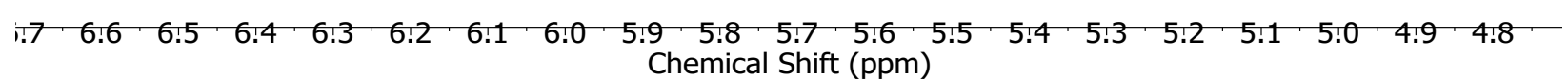

Figure S44. Multipeak fitting functions of the bound and free ligands shown here for UDT/oleate$\mathrm{PbS}$ QD with 500 eq. CoCp 2 added after 123 hours. The original spectrum is shown in the black trace, the fits are shown as blue traces with the sum shown as a pink trace. Bound oleate was fit to two peaks located at 5.65 and $5.58 \mathrm{ppm}$ and free oleate was fit to a single peak at $5.46 \mathrm{ppm}$. Bound UDT was calculated by averaging the broad peak at $5.98 \mathrm{ppm}$ (alkene $\mathrm{CH}, 1 \mathrm{H}$ ) and the two peaks at 5.20 and $5.12 \mathrm{ppm}$ (alkene $\mathrm{CH}_{2}, 2 \mathrm{H}$ ), normalized to the same number of protons. Free UDT was calculated by averaging the broad peak at $5.80 \mathrm{ppm}(1 \mathrm{H})$ and the four peaks between 5.07 and 4.97 ppm $(2 \mathrm{H})$, normalized to the same number of protons. The residual of the fit is shown in red. Peaks at $6.06 \mathrm{ppm}$ and the triplet at $4.93 \mathrm{ppm}$ are minor impurities. 


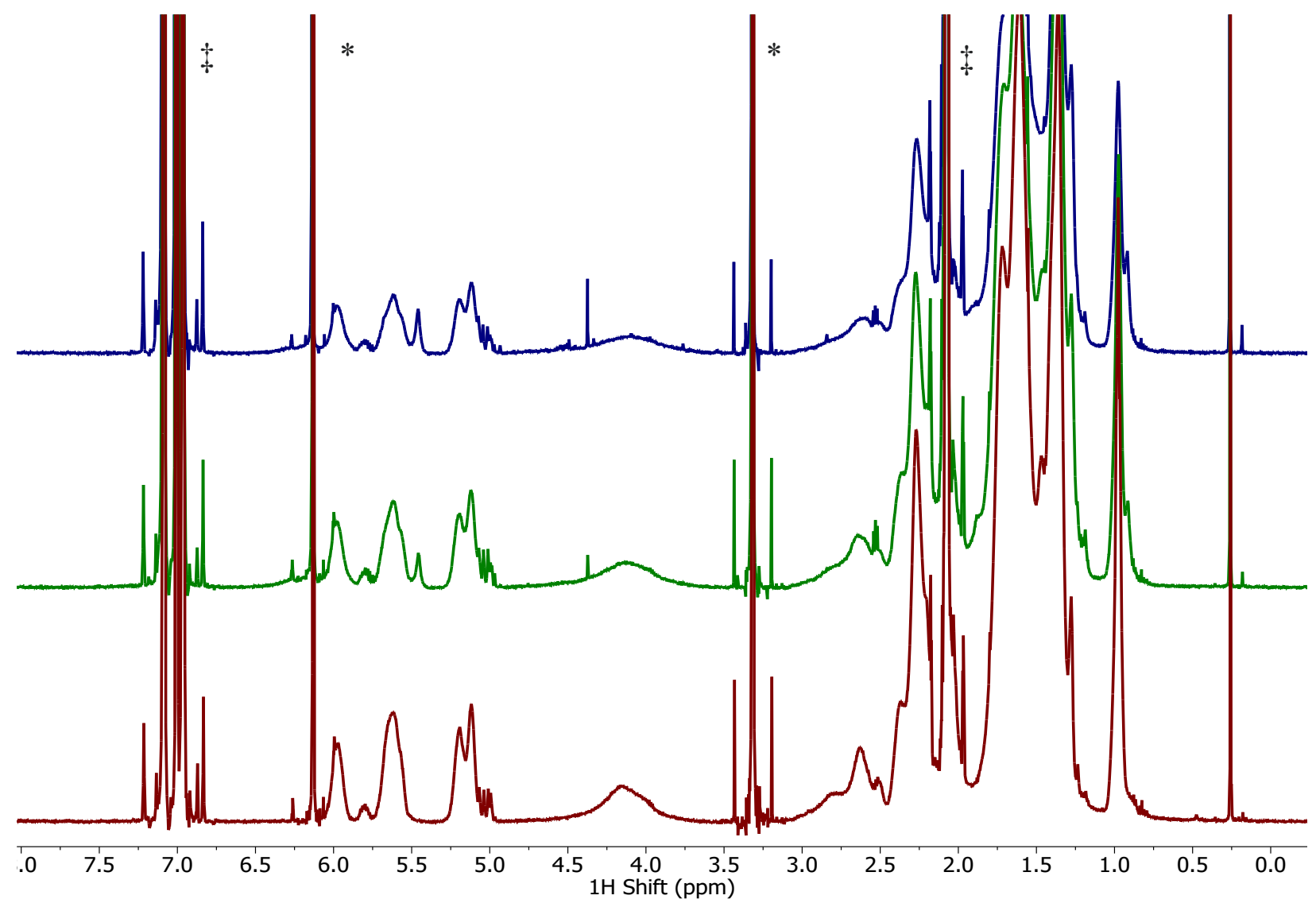

Figure S45. $600 \mathrm{MHz}{ }^{1} \mathrm{H}$ NMR spectra in toluene- $d_{8}$ of UDT/oleate-PbS QDs with 0 (red), 100 (green), and 500 (blue) eq CoCp 2 added after 123 hours. Peaks denoted by $\ddagger$ are toluene solvent residuals, and peaks marked by * are the internal standard 1,3,5-trimethoxybenzene. 


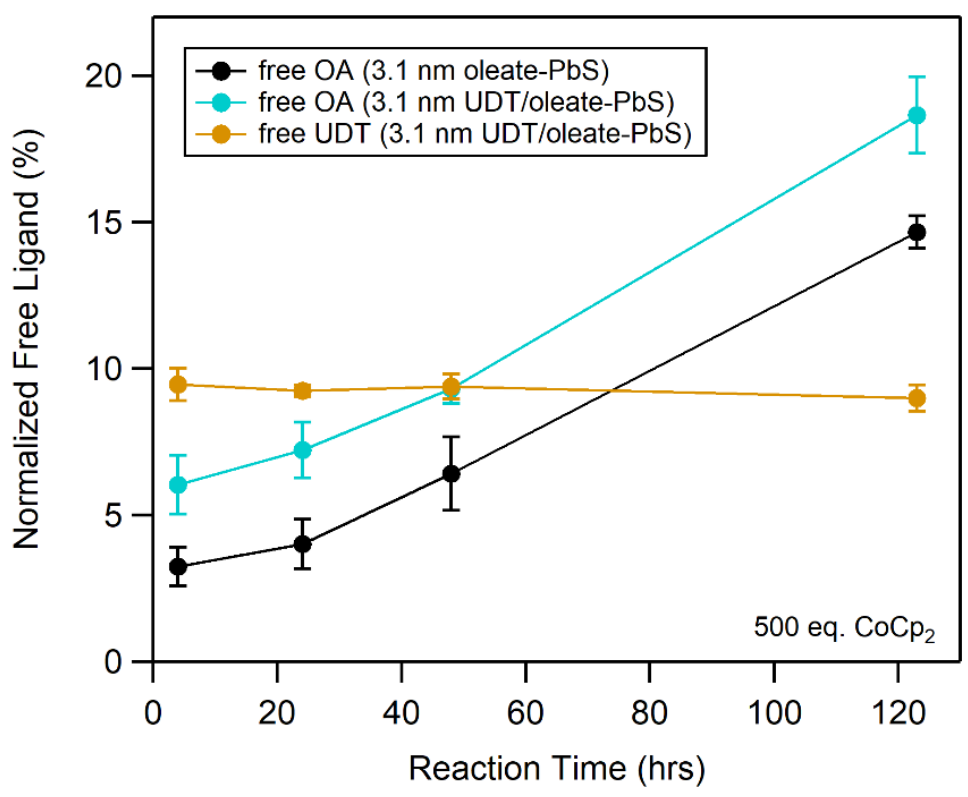

Figure S46. Quantification of free ligand in oleate-PbS-3 (black) and UDT/oleate-PbS (yellow, blue) batches with 500 eq $\mathrm{CoCp}_{2}$ added over time (average values from triplicate runs \pm standard deviation). Importantly, the amount of free UDT at time $=0$ remains consistent throughout the titration. Lines connecting data points are a guide to the eye.

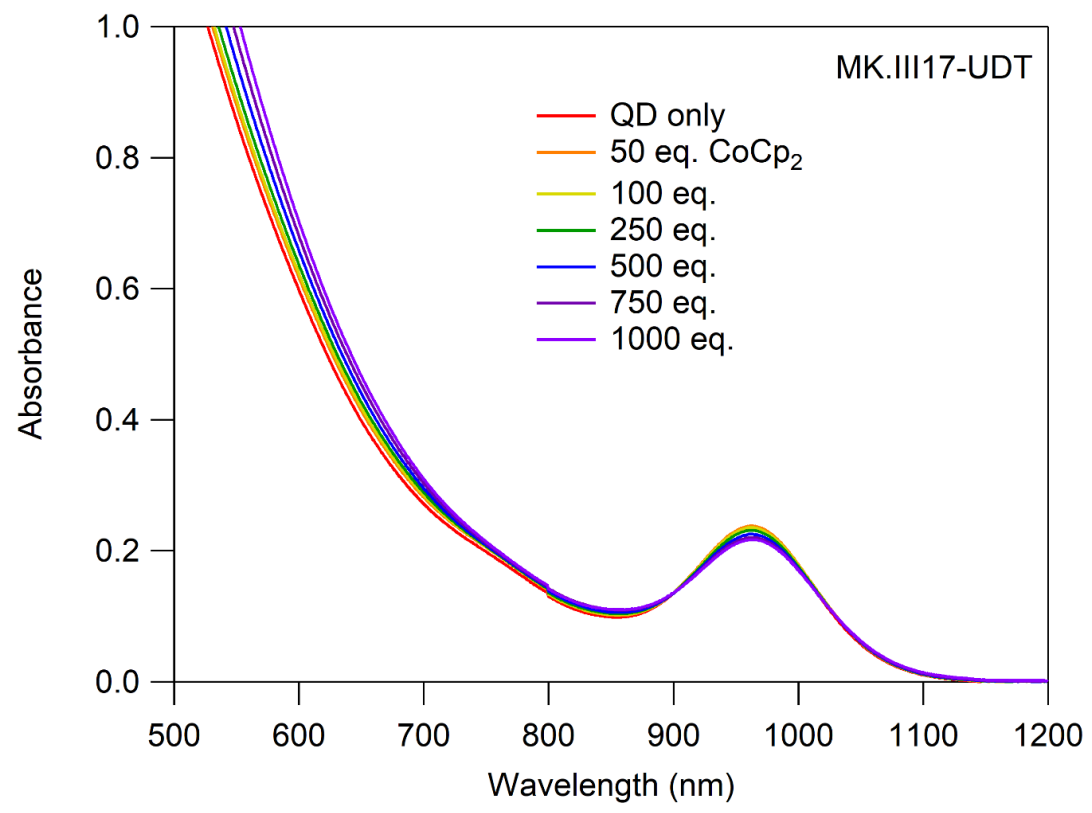

Figure S47. UV-Vis-NIR absorption spectrum of UDT/oleate-PbS in toluene $(2.5 \mu \mathrm{M})$ with added excess $\mathrm{CoCp}_{2}$. We observe a $1 \mathrm{~nm}$ red shift and minor loss of absorbance with added $\mathrm{CoCp}_{2}$. 


\section{Qualitative molecular orbital diagrams to illustrate $\mathbf{P b}^{2+}$-ligand energetics}
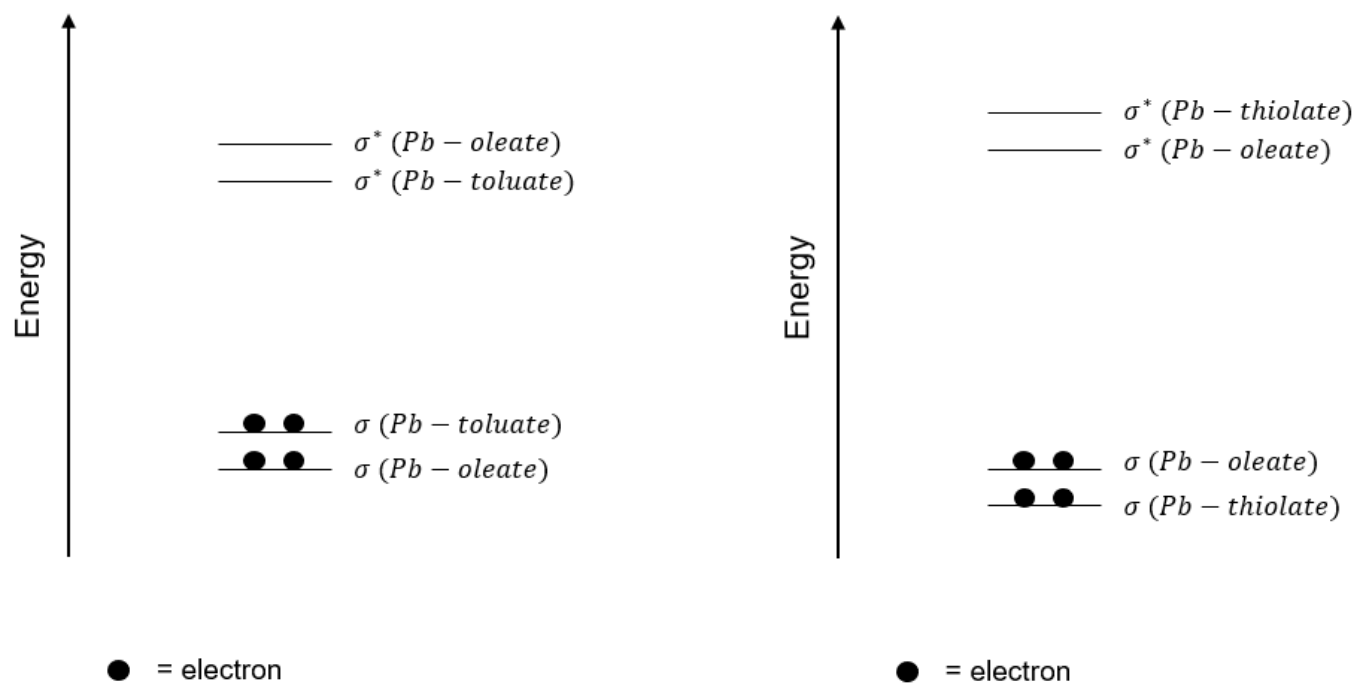

The qualitative molecular orbital (MO) diagram on the left shows the relative energetics of the bonding $(\sigma)$ and antibonding $\left(\sigma^{*}\right)$ orbitals formed between the ligands (toluate and oleate) and surface $\mathrm{Pb}^{2+}$ ions. Due to the weaker interaction between $\mathrm{Pb}^{2+}$ ions and toluate, the bonding $\mathrm{MO}$ of $\mathrm{Pb}^{2+}$-toluate is destabilized and the antibonding $\mathrm{MO}$ of $\mathrm{Pb}^{2+}$-toluate is stabilized, relative to the analogous MOs between $\mathrm{Pb}^{2+}$ ions and oleate. Conversely, the qualitative $\mathrm{MO}$ diagram on the right conveys the stabilization of the bonding $\mathrm{MO} \mathrm{of} \mathrm{Pb}^{2+}$-thiolate and the destabilization of the antibonding $\mathrm{MO}$ of $\mathrm{Pb}^{2+}$-thiolate compared with those of $\mathrm{Pb}^{2+}$-oleate. Although we have chosen to pin the energies of $\mathrm{Pb}^{2+}$-oleate molecular orbitals for comparison across the two diagrams, these diagrams are qualitative and the exact energies of each orbital are unknown and may differ between each mixed-shell QD system.

\section{REFERENCES}

(1) Giansante, C. Surface Chemistry Control of Colloidal Quantum Dot Band Gap. J. Phys. Chem. C 2018, 122, 18110-18116. 